\title{
Genome-Wide Analyses of Repeat-Induced Point Mutations in the Ascomycota
}

\author{
Stephanie van Wyk, Brenda D. Wingfield, Lieschen De Vos, Nicolaas A. van der Merwe \\ and Emma T. Steenkamp*
}

Department of Biochemistry, Genetics and Microbiology, Forestry and Agricultural Biotechnology Institute (FABI), University of Pretoria, Pretoria, South Africa

The Repeat-Induced Point (RIP) mutation pathway is a fungus-specific genome defense mechanism that mitigates the deleterious consequences of repeated genomic regions and transposable elements (TES). RIP mutates targeted sequences by introducing cytosine to thymine transitions. We investigated the genome-wide occurrence and extent of RIP with a sliding-window approach. Using genome-wide RIP data and two sets of control groups, the association between RIP, TEs, and GC content were contrasted in organisms capable and incapable of RIP. Based on these data, we then set out to determine the extent and occurrence of RIP in 58 representatives of the Ascomycota. The findings were summarized by placing each of the fungi investigated in one of six categories based on

Edited by:

Daniel Yero,

Autonomous University of Barcelona

Spain

Reviewed by:

Braham Dhillon,

University of Florida, United States Ursula Oggenfuss,

Université de Neuchâtel, Switzerland

*Correspondence:

Emma T. Steenkamp emma.steenkamp@fabi.up.ac.za

Specialty section:

This article was submitted to Evolutionary and Genomic Microbiology,

a section of the journal

Frontiers in Microbiology

Received: 28 October 2020 Accepted: 29 December 2020

Published: 01 February 2021

Citation:

van Wyk S, Wingfield BD, De Vos $L$, van der Merwe NA and Steenkamp ET (2021) Genome-Wide

Analyses of Repeat-Induced Point Mutations in the Ascomycota.

Front. Microbiol. 11:622368.

doi: 10.3389/fmicb.2020.622368 the extent of genome-wide RIP. In silico RIP analyses, using a sliding-window approach with stringent RIP parameters, implemented simultaneously within the same genetic context, on high quality genome assemblies, yielded superior results in determining the genome-wide RIP among the Ascomycota. Most Ascomycota had RIP and these mutations were particularly widespread among classes of the Pezizomycotina, including the early diverging Orbiliomycetes and the Pezizomycetes. The most extreme cases of RIP were limited to representatives of the Dothideomycetes and Sordariomycetes. By contrast, the genomes of the Taphrinomycotina and Saccharomycotina contained no detectable evidence of RIP. Also, recent losses in RIP combined with controlled TE proliferation in the Pezizomycotina subphyla may promote substantial genome enlargement as well as the formation of sub-genomic compartments. These findings have broadened our understanding of the taxonomic range and extent of RIP in Ascomycota and how this pathway affects the genomes of fungi harboring it.

Keywords: repeat-induced point mutation, RIP, Ascomycota, genome evolution, GC content

\section{INTRODUCTION}

The Ascomycota represents the largest taxonomic division of the fungal kingdom and includes a vast collection of diverse species (Schoch et al., 2009). These fungi are ubiquitous and can be found in all environmental settings where they are key players in the recycling of organic matter in aquatic and terrestrial systems (Mueller and Schmit, 2007). The group is also notorious for its pathogenic potential and many of them threaten not only human and animal health, but also all forms of plant life (Bowman et al., 1992; Dong et al., 2015). Apart from their 
medical and economic importance, certain Ascomycota are also exploited for their industrial and commercial properties (de Oliveira and de Graaff, 2011; Grigoriev et al., 2011). Their products are essential in fermentation processes and the production of sought after foods and products, such as meat alternatives (Kumar et al., 2017), biofuels (Grigoriev et al., 2011), organic chemicals (Payne et al., 2015), enzymes, and antibiotics (Martens and Demain, 2017).

The reproductive biology of the Ascomycota is remarkably diverse (Billiard et al., 2012; Wilken et al., 2017). Many reproduce sexually via meiosis to generate progeny in the form of ascospores. In such fungi, maintenance of this ability ensures the generation of genetic diversity and reshuffling of existing variation within populations. Sexual reproduction and meiosis also allow certain members of the phylum use of the Repeat-Induced Point (RIP) mutation pathway for genomic defense against "selfish" or repeated sequence elements (Selker and Garrett, 1988; Selker, 1990). The RIP pathway operates as a type of "quality control" mechanism that ensures integrity of the fungal genome by protecting it from the genetic consequences of these elements, particularly those associated with Transposable Elements (TEs; Cambareri et al., 1991; Selker, 2002). It distinguishes targets based on shared homology between duplicates (Selker and Garrett, 1988), where the targeted regions are permanently mutated by the introduction of cytosine to thymine transition mutations (Cambareri et al., 1991; Watters et al., 1999). Because the essential processes of the pathway are only active after fertilization and immediately before meiosis occurs (Selker and Garrett, 1988; Selker, 1990), RIP is considered to be exclusively available to fungi that are actively recombining.

The RIP pathway evolved early in the evolutionary history of fungi, most likely before the divergence of the subkingdom Dikarya (Horns et al., 2012; Meerupati et al., 2013). RIP has been experimentally and computationally observed only in Ascomycota and certain Basidiomycota (Clutterbuck, 2011; Horns et al., 2012; Hane et al., 2015). The exact taxonomic range of RIP remains ill-defined, but RIP mutation has been recorded in the early diverging lineages of the Pezizomycotina (Meerupati et al., 2013), as well as members of the classes Sordariomycetes and Dothideomycetes (Clutterbuck, 2011).

The study of RIP has allowed important conclusions to be drawn regarding the role of this process in fungal evolution. Apart from maintaining genome integrity, RIP can bring about a range of functionally consequential genetic changes (Rouxel et al., 2011; Meerupati et al., 2013; King et al., 2015; Testa et al., 2016; van Wyk et al., 2019a). This is because RIP activity can lead to the formation of long stretches of AT-rich sequences, causing such genomic regions to be generally gene sparse (Rouxel et al., 2011; van Wyk et al., 2019a). Also, large-scale accumulation of RIP products promotes the loss of sequence similarity between homologous DNA sequences (Ellison et al., 2011; van Wyk et al., 2019a), while RIP-associated methylation brings about suppressed recombination (Ellison et al., 2011). The combined effects of these processes contribute to lineage divergence and lineage-specific evolution, as have been observed in fungi such as Neurospora tetrasperma (Ellison et al., 2011; Sun et al., 2017) and species in the Fusarium fujikuroi complex (van Wyk et al., 2019a).

Since the discovery of RIP, a range of in silico methods have been used to study RIP (Hane and Oliver, 2008, 2010; Horns et al., 2012; Testa et al., 2016; van Wyk et al., 2019b). In these methods, RIP is identified based on the occurrence of extensive directional mutations (i.e., cytosine to thymine transition mutations; Clutterbuck, 2011). Genomic regions affected by RIP display increased frequencies of transition to transversion ratios, and further exhibit reduced GC content (Hane and Oliver, 2008; Clutterbuck, 2011). In silico RIP analyses also account for the genetic context of RIP transitions, where RIP detection is dependent on the nucleotide sequences adjacent to those recognized as appropriate RIP targets (Selker et al., 2003). Therefore, the occurrence of cytosine to thymine biased transitions, together with the genetic context of these mutations, provided the theoretical basis for the commonly used RIP indices (Margolin et al., 1998; Selker et al., 2003; Lewis et al., 2009; van Wyk et al., 2019b).

Despite the availability of different in silico techniques for studying RIP, little is known about the occurrence and extent of RIP on a genome-wide level for the Ascomycota. This is mainly because of the technical complexities associated with the detection of RIP, which often relies on appropriate alignment of homologous genomic segments, especially those containing TEs and repeated regions (Hane and Oliver, 2008). However, accurate identification of these elements is dependent on the comprehensiveness of existing TE databases, while the elements in question could have undergone extensive degradation since their initial emergence (Hane et al., 2015). Also, similar types of repeats and TEs may not be present in all the taxa investigated, which further hinders the analysis of RIP across a broad taxonomic range. Although sliding window-based approaches have been previously established, these have not been adopted widely due to the extensive occurrence of false positive results associated with the use of overly lenient parameters (Hane and Oliver, 2008; van Wyk et al., 2019b). To exacerbate the matter further, most analyses of RIP are performed within the bounds of gene-level duplications or TE repeats (Hane and Oliver, 2008) and do not take into account the fact that RIP may "leak" beyond the bounds of genetic targets, thereby affecting adjacent genomic regions (Irelan et al., 1994; Rouxel et al., 2011; Van de Wouw et al., 2018; van Wyk et al., 2019a). The consequence of all these complexities and limitations is that our understanding of the extent of RIP on a genome-wide scale remains unclear, because such information cannot be readily extrapolated from TE and gene-level RIP analyses.

This study considered the genome-wide effects of the RIP pathway in the Ascomycota using whole genome sequences available in the public domain. To overcome the typical challenges associated with RIP analyses, we used an alignment-free method, based on a sliding-window approach with optimized RIP index parameters (van Wyk et al., 2019b) rather than single-type TE/repeat sequence matrices (Hane and Oliver, 2008). Our three specific aims were to (i) evaluate the extent to which genome quality might influence the detection of RIP; (ii) establish baseline criteria for recognizing 
RIP capability in fungal genomes using in silico analysis; and (iii) compare the distribution and extent of RIP across the genomes of Ascomycota. The results of this study will provide valuable insights regarding the taxonomic range of RIP and how it affects the genomes of the fungi harboring it.

\section{MATERIALS AND METHODS}

\section{Genome Sequences}

Publicly available whole-genome sequences were obtained from the database of the National Centre for Biotechnology Information (NCBI). These included sequenced representatives of all subphyla of the Ascomycota, i.e., Pezizomycotina (46 genomes), Saccharomycotina (nine genomes), and Taphrinomycotina (three genomes). The repeat content for each genome was estimated using the REPET pipeline (Quesneville et al., 2005; Flutre et al., 2011). Based on reports from published literature, the fungi in our data set were also assigned as "sexual," "asexual," and "unknown" (Supplementary Table S1, and associated references), because RIP is considered to be associated with premeiotic processes (Selker and Garrett, 1988; Selker, 1990). Where relevant, RIP-positive and RIP-negative control genome sequences were included (Supplementary Table S1, as described previously; van Wyk et al., 2019b). These controls included six genomes of organisms that are, respectively, RIP-capable (i.e., Neurospora crassa, Trichoderma reesei, and Leptosphaeria maculans; Selker et al., 2003; Fudal et al., 2009; Li et al., 2017) and not RIP-capable (i.e., Escherichia coli, Encephalitozoon cuniculi, and Candida albicans; Supplementary Table S1).

Representatives for each taxonomic group were chosen based on the quality and completeness of their genome assemblies. Where possible, representatives classified as "complete" or "chromosome" assembly level, according to the NCBI database, with high N50 values were also selected for subsequent analyses (Supplementary Table S1). All taxa investigated were evaluated with the Benchmarking Universal Single-Copy Orthologs (BUSCO) v. 3.0.2 (Simão et al., 2015; Waterhouse et al., 2017) as a further indication of genome completeness using the following data sets: Saccharomyceta, Pezizomycotina, Ascomycota, and Microsporidia.

To investigate how the quality and completeness of a genome assembly influences genome-wide RIP statistics, three versions of the Fusarium circinatum genome (strain FSP34) were subjected to RIP analysis (Wingfield et al., 2012, 2018; van Wyk et al., 2019a) and the identification of Large RIP-Affected Regions [LRARs; i.e., RIP-affected regions spanning at least 4,000 base pairs (bp); van Wyk et al., 2019b]. This fungus was originally isolated from pitch canker affected Pinus radiata tissue in California (Wingfield et al., 2012) and is maintained at $-80^{\circ} \mathrm{C}$ in the culture collection of the Forestry and Agricultural Biotechnology Institute (FABI; University of Pretoria, Pretoria, South Africa). These three assembly versions represent improvements over time as new technologies and more data were used for determining the genome sequence of this fungus. They are referred to as the 2012, 2018, and 2020 versions of the F. circinatum (FSP34) genome assembly (Supplementary Table S1).
Additionally, the level of genome completeness of the three F. circinatum genome assemblies was verified using the BUSCO tool with the Sordariomyceta data set (Simão et al., 2015; Waterhouse et al., 2017).

\section{Establishing Baseline Criteria for Recognizing RIP Capability Using in silico Analysis}

Because no previous studies have set out to quantitatively determine the genome-wide occurrence of RIP among members of the Ascomycota, we first needed to determine a baseline to contrast and distinguish fungi that potentially have RIP and those that do not. To this effect, we considered two approaches. The first was to determine whether a particular fungal genome encoded all of the expected genes associated with RIP-capability, DNA methylation, and RIP directed heterochromatic silencing, and the second was whether it contained RIP-like transition mutations. For this purpose, we used a set of positive and negative control genomes.

To study the presence and distribution of genes associated with the functioning of RIP (based on reports from N. crassa; Lewis et al., 2010; Gessaman and Selker, 2017; Gladyshev, 2017; Li et al., 2017; Bewick et al., 2019; van Wyk et al., 2019a; He et al., 2020), BLASTp and tBLASTn searches were performed on the fungi investigated in this study. DNA and protein sequences with sufficient similarity (Expectvalue $<1 \times 10^{-5}$ ) to those described in $N$. crassa were identified using the NCBI non-redundant BLAST database. Although the exact genomic mechanism underlying RIP is still under investigation, the query sequences used in these analyses were the two 5-cytosine methyltransferases RID (RIP deficient) and defective in methylation (DIM-2). In these analyses, we also included six other genes whose products were previously suggested to play a role in processes related to DIM-2-mediated DNA methylation and RIP directed heterochromatic silencing in the Ascomycota. These were the cofactors heterochromatic protein 1 (HP-1), DIM-5, DIM-7, DNA damage binding protein 1 (DDB-1), and Cullin-4 (Cul4; Lewis et al., 2010; Gessaman and Selker, 2017; He et al., 2020).

To study the distribution of RIP, and to evaluate the most informative and suitable method for identifying RIP within fungal genomes, the six control organisms used in this study were subjected to sliding window-based RIP analyses using two tools: RIPCAL (v1.0.4) and The RIPper (Hane and Oliver, 2008; van Wyk et al., 2019b). In addition to full RIP analyses as described previously (van Wyk et al., 2019b), we also used The RIPper to investigate the occurrence of RIP mutations relative to changes in GC content for individual windows of each of the control genome sequences. For this, RIP composite indices and GC content were calculated. In all cases, we used 1,000 bp sliding windows and $500 \mathrm{bp}$ step sizes (van Wyk et al., 2019b).

We also complemented the RIP data generated for the control organisms with information on the distribution of TEs obtained from REPET. For Le. maculans, chromosome-sized scaffolds were not available, and the 10 largest scaffolds were investigated. 
Because N. crassa is the model organism for RIP (Gladyshev, 2017), detailed genome-wide RIP analyses were performed on each of its chromosomes (linkage groups) to evaluate the occurrence of RIP in telomeric and centromeric regions. To this end, the frequency of telomeric repeat sequences were determined (Lewis et al., 2009; Wu et al., 2009; van Wyk et al., 2018) using a $100 \%$ similarity cut-off value for the “TTAGGG" telomeric repeat sequence. Putative genomic regions corresponding to centromeres were identified based on the approach reported previously (Li et al., 2017; i.e., identification of the longest AT-rich blocks in each N. crassa chromosome). To determine the proportion of TEs distributed within N. crassa LRARs, REPET annotations, and CLC Genomics Workbench v. 8.0 (CLCbio, Aarhus, Denmark) were used.

For comparative purposes, the information generated using the REPET pipeline for the control organisms were used for alignment-based RIP analyses (Hane and Oliver, 2008). The TE data sets were constructed by aligning TE repeat sequences sharing a high degree of nucleotide similarity to Large Retrotransposon derivative (LARD) TE sequences, which were identified using BLASTn searches in CLC Genomics Workbench (Expect-value $<1 \times 10^{-5}$ ). The resulting data sets were aligned using the multiple sequence alignment online tool MAFFT (v7.471; Katoh et al., 2019), after which each data set was subjected to alignment-based RIP analyses and dinucleotide frequency analyses using highest GC content consensus implemented in RIPCAL (Hane and Oliver, 2008).

By making use of the control genomes, we also investigated the possibility of erroneous identification of RIP using 100 simulated sequences of $100,000 \mathrm{bp}$ in length. These were generated with the Random DNA sequence tool (Stothard, 2000) and subjected to full RIP analyses as before. A chi-square test was used to evaluate whether the frequency of RIP-positive windows in the simulated data and that of the genome sequences investigated differed significantly $(p<0.01)$. For these analyses, the null expectation was that the total number of RIP-positive windows in a particular genome assembly is the same as in the simulated data set.

\section{Genome-Wide in silico RIP Analysis of the Ascomycota}

Whole-genome sequence information of species included in this study (Supplementary Table S1) was subjected to RIP analyses using the RIPper (van Wyk et al., 2019b). These analyses employed the stringent RIP parameters (i.e., RIP product $>1.15$, RIP substrate $\leq 0.75$, and a RIP composite index $>0$ ) suggested previously (van Wyk et al., 2019b). Possible correlations in our datasets (e.g., genomic proportion containing repetitive sequences vs. those containing RIP mutations) were tested using the Free Statistics and Forecasting Software v1.2.1 (Wessa, 2020).

The results of the occurrence and extent of RIP were summarized, and these data were used to categorize individual genomes in different RIP classes. Class 1 referred to genomes containing no RIP $(0.0$ to $<0.2 \%)$, Class 2 for genomes containing trace levels of RIP (total genomic proportion between 0.2 and $<1.0 \%$ ), Class 3 moderately low RIP (total genomic proportion 1.0 to $<5.0 \%$ ), Class 4 moderate RIP (total genomic proportion 5.0 to $<10.0 \%$ ), Class 5 moderately high RIP (total genomic proportion 10.0 to $<20.0 \%$ ), and Class 6 high RIP (total genomic proportion $\geq 20.0 \%$ ). Additionally, we determined the presence and distribution of genes encoding DIM-2 and RID together with the five cofactors associated with DIM-2mediated DNA methylation across the selected Ascomycota. This was done as described above for the control genomes. Finally, we also evaluated the possibility of erroneous identification of RIP in these fungal genomes using simulated data and chi-squared tests as described above. The results obtained for all these analyses were illustrated with a cladogram reflecting the phylogenetic relationships among the taxa included using their taxon classification. The cladogram was constructed in phyloT using NCBI taxonomic identification numbers and visualized using iTOL v. 3 software (Letunic and Bork, 2016).

\section{RESULTS}

\section{RIP Analyses, Genome Completeness, and Quality of the Genome Assemblies}

To evaluate the influence of assembly quality and genome completeness on the genome-wide RIP statistics obtained for a particular organism, three versions $(2012,2018$, and 2020) of the genome assembly for F. circinatum isolate FSP34 were used (Table 1). These assemblies differed considerably in size, number of contigs/scaffolds, and BUSCO completeness level. The RIPper-based analyses of these assemblies further showed that the total proportion of each assembly that constituted RIP mutations were $4.0,4.1$, and $6.3 \%$, respectively. In other words, with an improvement in genome quality, more RIP mutations could be detected. The same was also true for the total proportion predicted repeat content ( 4.6 vs. $8.0 \%$ for the 2012 and 2020 assembly versions) and number of LRARs detected (45 vs. 168 for the 2012 and 2020 assembly versions). These data thus show that the effects of RIP in a particular fungal genome would be underestimated if low-quality genome sequences are used in the analysis.

Due to the substantial impact of genome quality on detection of the substrates and products of RIP, we attempted to exclude genomes of low-quality (as reflected by high numbers of scaffolds) or low completeness (as indicated by a BUSCO completeness) in our subsequent analyses. Accordingly, we included 59 fungal genomes for the study (Supplementary Table S1). Many of the genomes with high BUSCO completeness values were generally also assembled to "chromosome-level" (according to the NCBI database), or when these were not available, to "scaffold-level" (Supplementary Tables S1 and S2). For example, among the 59 fungal genomes, 46 contained $96-99.7 \%$ complete BUSCOs and only 11 contained $91-95 \%$ complete BUSCOs. Despite their lower completeness, the latter genomes were included in the analysis because they were classified as complete by NCBI and contained a small number of scaffolds (Supplementary Table S1), suggesting that their reduced BUSCO values might be a consequence of their unique evolutionary histories (Simão et al., 2015). 
TABLE 1 | Genome-wide Repeat-Induced Point (RIP) mutation statistics for different versions of the Fusarium circinatum (FSP34) genome assembly.

\begin{tabular}{|c|c|c|c|}
\hline \multirow{2}{*}{ Genome and RIP statistics } & \multicolumn{3}{|c|}{ Version of the genome assembly } \\
\hline & 2012 & 2018 & 2020 \\
\hline Genome size (bp) & $42,457,838$ & $43,949,211$ & $45,100,144$ \\
\hline $\begin{array}{l}\text { Number of scaffolds/contigs in the } \\
\text { genome assembly }\end{array}$ & 4,509 & 420 & 28 \\
\hline BUSCO genome completeness $(\%)^{1}$ & 91.1 & 92.3 & 98.1 \\
\hline Number of windows investigated & 88,515 & 87,898 & 90,200 \\
\hline GC content (\%) for the assembly & 47.3 & 47.4 & 46.9 \\
\hline Number of RIP affected windows ${ }^{2}$ & 3,582 & 3,628 & 5,685 \\
\hline RIP-affected genomic proportion $(\%)^{3}$ & 4.0 & 4.1 & 6.3 \\
\hline Predicted repeat content $(\%)^{4}$ & 4.6 & 4.3 & 8.0 \\
\hline Number of LRARs ${ }^{5}$ & 45 & 106 & 168 \\
\hline Average size (bp) of LRARs & 7085.4 & 9547.2 & 14975.5 \\
\hline Average GC content (\%) of LRARs & 22.8 & 22.2 & 20.8 \\
\hline Genomic proportion (bp) of LRARs & 318,842 & $1,012,006$ & $2,515,882$ \\
\hline Product index value for LRARs $^{6}$ & 1.6 & 1.6 & 1.6 \\
\hline Substrate index value for LRARs ${ }^{7}$ & 0.3 & 0.3 & 0.2 \\
\hline Composite index value for $\mathrm{LRARs}^{8}$ & 1.3 & 1.3 & 1.3 \\
\hline $\begin{array}{l}{ }^{1} \text { Benchmarking universal single-copy orth } \\
\text { 2017). } \\
{ }^{2} \text { Based on RIP index values. } \\
{ }^{3} \text { Proportion of the genome affected by RIl } \\
\text { windows indicating RIP-positive index vall } \\
\text { investigated for the entire genome sequer } \\
{ }^{4} \text { Estimated using REPET (Quesneville et a } \\
{ }^{5} \text { LRAR, large RIP affected genomic region } \\
\text { RIP affected. } \\
{ }^{6} \text { Product index value (TpA/ApT): } x>1.15 \text {. } \\
{ }^{7} \text { Substrate index value }(C p A+T p G / A p C \\
{ }^{8} \text { Composite index value }[(T p A / A p T)-(C p\end{array}$ & $\begin{array}{l}\text { Calculated us } \\
\text { les against the } \\
\text { ce. } \\
\text { 2005; Flutre } \\
\text { s. More than 4, }\end{array}$ & $\begin{array}{l}\text { ing the total nun } \\
\text { total number of } \\
\text { ooo bp that are }\end{array}$ & rhouse et al., \\
\hline
\end{tabular}

The remaining three genomes selected had exceedingly low BUSCO completeness scores (Supplementary Tables S1 and S2) and were for Pichia kudriavzevii (BUSCO: 80.0\%), Duddingtonia flagrans (BUSCO: 89.1\%), and Symbiotaphrina buchneri (BUSCO: $75.6 \%)$. They were retained for the current study because the published assembly for Pi. kudriavzevii represents the complete genome, sequenced from chromosome telomere to telomere (Douglass et al., 2018). The other two apparently incomplete genomes were included to ensure taxonomic breadth (i.e., representatives from the Orbiliomycetes and Xylonomycetes, respectively).

\section{Establishing Baseline Criteria for Recognizing RIP Capability Using in silico Analysis}

For establishing criteria with which to infer whether or not a particular fungus is potentially RIP-capable, we utilized the set of positive and negative control genomes. BLAST analyses against these genomes revealed that the positive controls encoded the two methyltransferases (DIM-2 and RID) demonstrated to mediate the RIP pathway in N. crassa (Supplementary Table S3; Figure 1). They also encoded the cofactors associated with DIM-2-mediated DNA methylation (Lewis et al., 2010; Gessaman and Selker, 2017; He et al., 2020), including DIM-5, DIM-7, Cul4, DDB-1, and HP-1 (Supplementary Table S3). None of the negative control fungal genomes encoded a full set of these seven genes.
The RIP statistics generated with The RIPper were consistent with previous work (van Wyk et al., 2019b), where considerable proportions of the positive control genomes had RIP mutations. Although analyses with RIPCAL produced similar results for the RIP-capable fungi, RIPCAL produced spurious results for the negative control genomes. While the statistics generated with The RIPper showed that all three of the negative control genomes were essentially devoid of RIP-like mutations (see below), the RIPCAL data suggested that considerable proportions of these genomes constituted RIP mutations (Supplementary Table S4). As pointed out before (Hane and Oliver, 2008), this discrepancy was likely due to lack of stringency in the parameters used for the sliding window-based RIP analyses tool implemented in RIPCAL.

Overall, the genome-wide RIP statistics generated with The RIPper showed that considerable proportions of the positive control genomes constituted RIP mutations, which was reflected in the number of RIP-affected windows showing high RIP composite index values and reduced GC content (Supplementary Table S4). The negative control organisms remained unchanged by RIP (van Wyk et al., 2019b), with the only exception being the microsporidian genome in which we detected a single RIP-affected window. Another striking difference between these groups was the complete lack of LRARs in any of the negative control organisms as was also observed previously (van Wyk et al., 2019b). Also, when the values for the composite RIP index were considered separate from other RIP index parameters, these values were generally very low and correlated with high GC content (Supplementary Table S4), confirming that they are unlikely to be due to RIP.

We also compared the relationship among TEs and repeated sequences, GC content, and RIP (Figure 2; Supplementary Figure S1). For the positive control fungi, changes in RIP composite index-values corresponded with drastically reduced GC content. This trend was also observed in genomic regions that displayed an increased frequency of TEs and repeats across individual chromosomes or scaffolds of the positive control genomes. By contrast, the distribution of TEs and repeats did not show the same interdependence with RIP composite index values and GC content in the negative control organisms. Notably, the changes in GC content of the negative control organisms remained relatively unchanged across the length of a chromosome or scaffold and did not have regions of extensive GC depletion.

Similar trends were observed when we performed alignmentbased and dinucleotide frequency RIP analyses for each of the LARD retrotransposon data sets constructed for the control genome assemblies (Supplementary Figure S1). For the RIP-capable fungi, RIP product, substrate, and composite index values indicated strong RIP responses. This was contrasted to the results obtained for the negative control organisms where changes in RIP index values did not indicate RIP in the TE data sets. Additionally, alignment-based RIP analyses showed that the genomes of RIP-capable fungi were characterized by high frequencies of cytosine to thymine transition mutations, where the dominant RIP mutation forms 


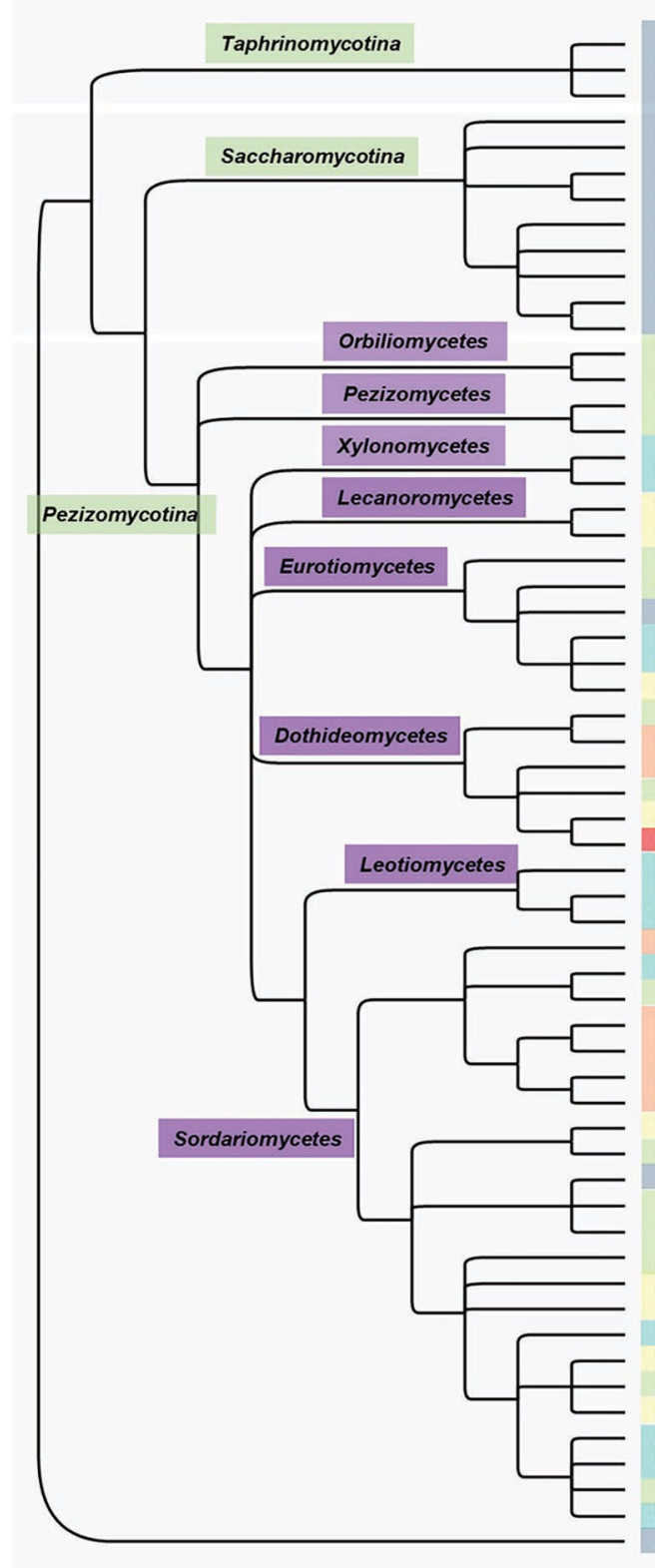

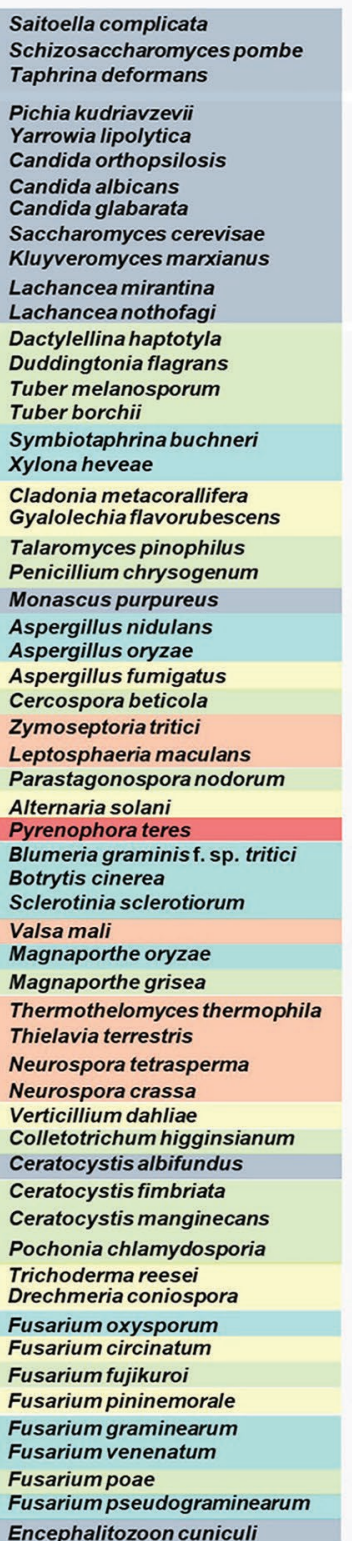

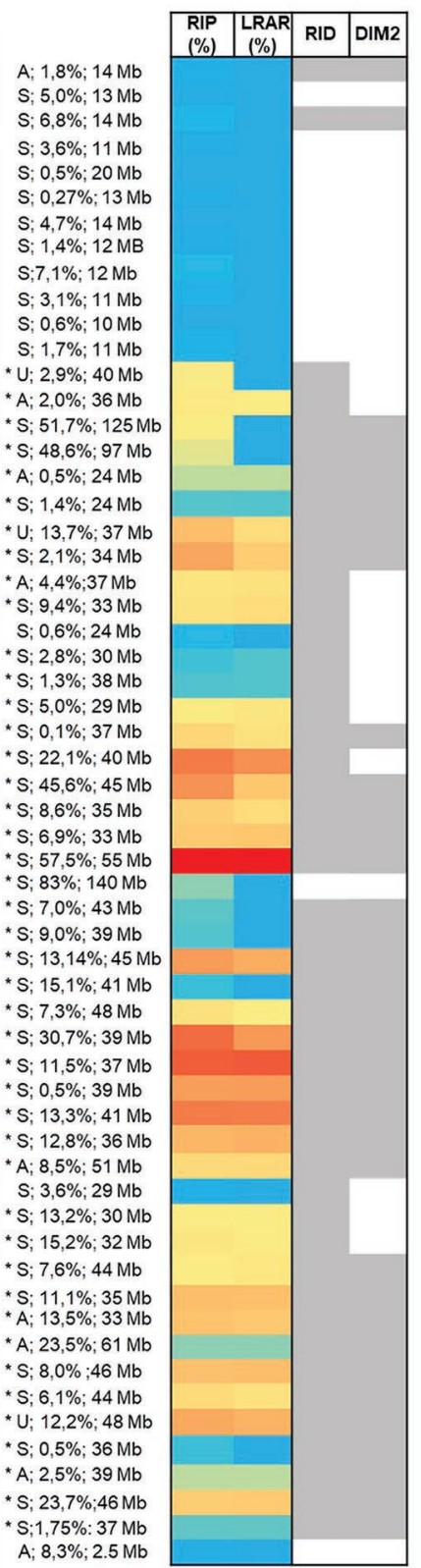

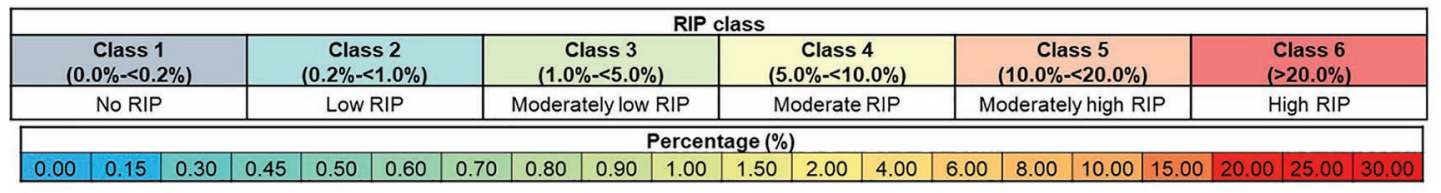

FIGURE 1 | Summary of genome-wide RIP analyses of Ascomycota investigated in this study. From left to right, the first panel represents a cladogram that summarizes the phylogenetic relationships among the fungi investigated, with the microsporidian fungus En. cuniculi serving as the root. An asterisk following species names denotes species where a chi-square test indicated that the frequency of RIP-positive windows in the simulated data and that of the actual genome studied differed significantly ( $p<0.01$ confidence level). The panel following the asterisk shows reproductive strategy ("A," "S," and "U," indicating asexual, sexual, and unknown), the predicted proportion (\%) of the genome that is repetitive, and genome size [Megabases (Mb)]. The next panel represents a heatmap that illustrates the total proportion (\%) of each genome assembly that constitutes RIP mutations and large RIP affected regions (LRARs). The last panel (right) indicates the presence/absence of the RIP-associated genes RID and DIM-2 in each genome (for the latter, see Supplementary Table S3 and Supplementary Figure S1 for detail about these two genes, as well as those for other RIP-associated genes), where shaded columns indicate that a putative homologous sequence identified. The color coding used in the different panels is explained in the keys provided. 


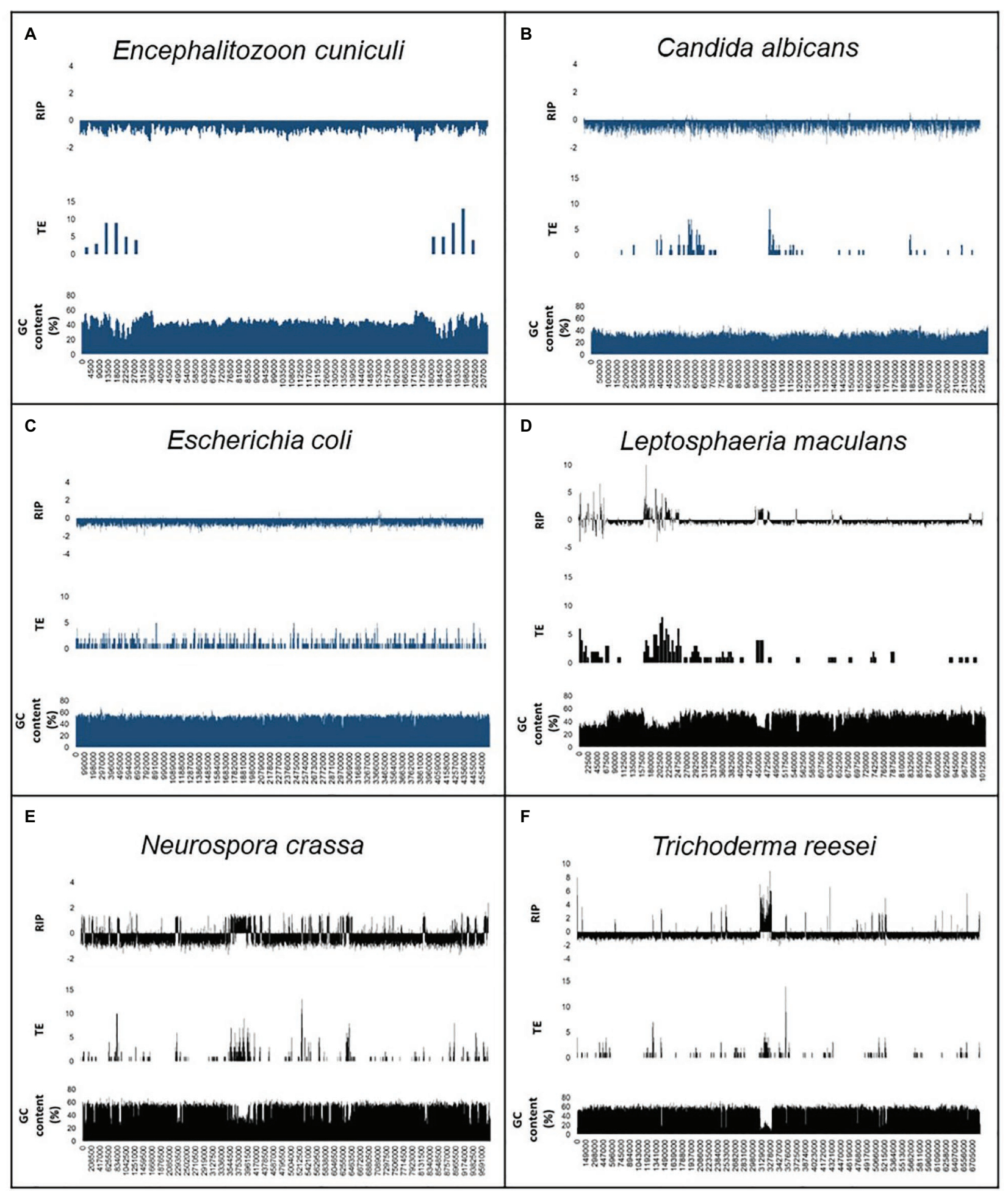

FIGURE 2 | Summary of the genomic features for the RIP-negative (A-C) and RIP-positive (D-F) control organisms used in this study. The first scaffold or chromosome is indicated for En. cuniculi (A), Ca. albicans (B), Le. maculans (D), N. crassa (E), and Tr. reesei (F), while the whole chromosome of E. coli (C) are shown. In each panel, the first row shows changes in RIP composite index values, where values $>0$ indicate RIP. The second and third rows show the number of transposable elements [TEs; calculated for every 10 and 5 kilo base pair (Kbp) increments] and GC content (calculated for every 1,000 and 500 bp increments). A full list of genome-wide RIP statistics, implementing stringent parameters, is available for these organisms in Supplementary Table S5. Results for the negative control organisms are indicated in blue, and positive control fungi are indicated in black. 
were CpT to TpT and ApG to ApA RIP transitions. Taken together, RIP was prominent in TE and repeat sequences of fungi known to be RIP-capable and absent in the TEs and repeats of organism not able to undergo RIP. These analyses were thus in agreement with those generated using a slidingwindow based approach implemented with The RIPper software. In other words, data generated using the RIPper allow for similar conclusions to be drawn regarding the presence of RIP in a representative assembly, but the RIPper additionally also provides quantitative insight on the extent, and the genomic location of RIP.

To further investigate regional variation in the occurrence and extent of RIP across the length of a chromosome, detailed RIP analyses were performed for the $N$. crassa chromosome assemblies (Figure 3; Supplementary Figure S2). The most extensively RIP-affected regions of the $N$. crassa chromosomes corresponded to that of the putative centromeric regions (Li et al., 2017). In addition, RIP- and TE-associated repeats were particularly prominent toward the ends of the chromosome, which generally corresponded to an overall increase in the density of telomeric repeat sequences. Therefore, regional variation in RIP across the length of the N. crassa chromosomes was common in telomeric and centromeric regions of chromosomes, as well in regions with TEs and repeated sequences.
Additionally, a substantial proportion (69.9\%) of the predicted TEs and TE repeated sequences were located within the LRARs.

Finally, we evaluated the possibility of erroneously identifying RIP in a particular fungal genome by contrasting the genomewide RIP statistics obtained with those for simulated data. In the simulated data sets, the frequency of windows with RIP was 0.095 and the frequency of windows without RIP was 99.905. However, for the positive control genomes, the frequencies of windows with RIP were $6.5,11.8$, and 14.5, while those without RIP were 93.5, 88.2, and 85.5 for Tr. reesei, N. crassa, and Le. maculans, respectively. Based on chi-square tests of independence, we thus rejected the null expectation that the frequencies for windows with and without RIP is the same as for the pattern seen with the simulated data sets, at a 99.99\% confidence level $(p<0.01)$ for each of the positive control genomes (Supplementary Table S5). However, the null expectation could not be rejected for the negative control En. cuniculi (Figure 1; Supplementary Table S5) suggesting that the one window containing RIP occurred by chance and was not a consequence of the RIP activity.

Based on the data generated using the set of control genomes, we considered a RIP-capable fungus as one in which RIP-like mutations constitute $>0.2 \%$ of its genome and the majority of RIP-positive windows have composite RIP index

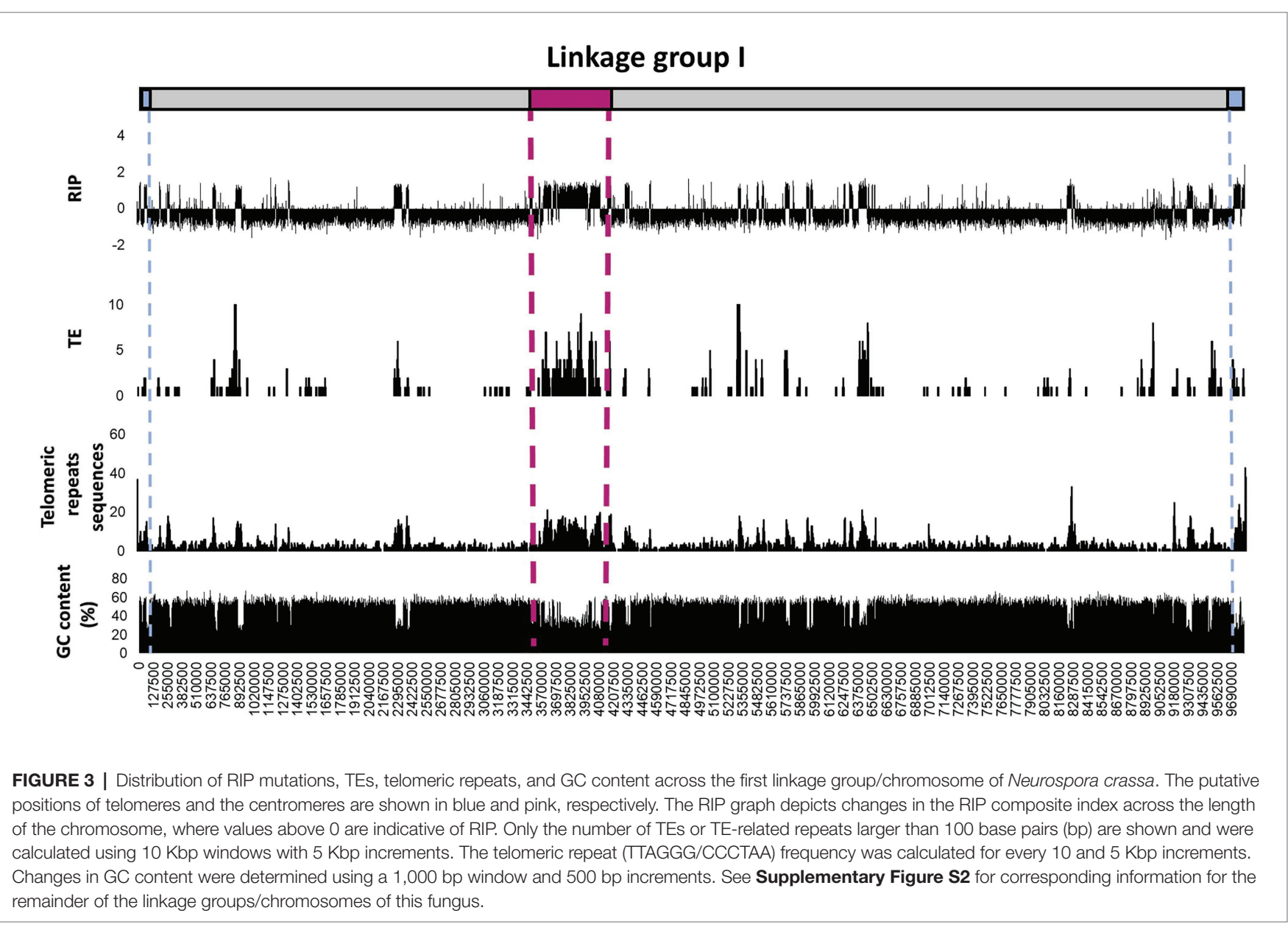


values higher than 1. The genomes of RIP-capable fungi are expected to also contain LRARs. Significant features of a genome containing RIP has correspondence between areas containing RIP mutations and reduced GC content compared to that of the remainder of the genome. For RIP-capable fungi, we further expect RIP to be a prominent feature of genomic regions rich in TE and other repeated sequences. Also, the genomes of such fungi would likely also maintain the RIP-associated genes, especially those encoding either or both of the 5-cytosine methyltransferases, RID, and DIM-2 (Gladyshev, 2017).

\section{Genome-Wide in silico RIP Analysis of the Ascomycota}

BLAST analyses with the sequences of the two methyltransferases known to mediate the RIP pathway, revealed that most of the genome assemblies for Pezizomycotina contained sequences with an average of 44 and 58\% similarity to, respectively, DIM-2 and RID from N. crassa (Supplementary Table S3; Figure 1). The only exceptions were Blumeria graminis f. sp. tritici lacking a copy of both genes, and representatives of the Orbiliomycetes, Ceratocystidaceae, and Zymoseptoria tritici lacking sequences with similarity to DIM-2. Two species from the Taphrinomycotina (i.e., Saitoella complicata and Taphrina deformans) also contained both genes. Genes encoding DIM-2 and RID were not detected in any of the other genomes, including all of the Saccharomycotina and En. cuniculi.

Regarding the cofactors associated with DIM-2-mediated DNA methylation (Lewis et al., 2010; Gessaman and Selker, 2017; Bewick et al., 2019; He et al., 2020), most of the genomes contained two or more of the five genes examined (Supplementary Table S3). All had sequences similar to those for DIM-5 and Cul4. However, all representatives of the Saccharomycotina lacked sequences for DDB-1 and HP-1, while
Bl. f. sp. tritici, was notable as the only representative of the Pezizomycotina lacking HP-1. In terms of DIM-7, genomes of En. cuniculi, Taphrinomycotina, Saccharomycotina, and 12 species in the Pezizomycotina (i.e., all representatives of Orbiliomycetes, Pezizomycetes, Leotiomycetes, and the Eurotiomycetes excluding Penicillium chrysogenum) lacked sequences encoding this cofactor.

To investigate the occurrence and extent of RIP in the genomes of Ascomycota, all of the genomes included in this study were subjected to genome-wide RIP analyses using The RIPper's sliding-window approach (Table 2; Supplementary Table S5; Figure 1). Plotting the results on a cladogram reflecting known phylogenetic relationships (McLaughlin et al., 2009) revealed that these fungi vary greatly in the total proportion of their genomes affected by RIP. While many harbored no evidence of RIP, others were rich in RIP. Among the Sordariomycetes and Dothideomycetes, some were highly affected by RIP, with the most extreme case being Pyrenophora teres where RIP constituted $29.53 \%$ of its genome. Genomes affected by RIP were also rich in LRARs (Figure 1). In those that were highly RIP-affected, many LRARs were recorded (e.g., as many as 439 LRARs in $Z$. tritici). In such cases, LRARs also constituted considerable proportions of the genomes examined, e.g., >16 Mbp of the 32.7 Mbp. genome of $P y$. teres. These LRARs further also corresponded to regions with reduced GC content (Table 2).

Overall, large variations were observed in terms of overall repeat content and genome size relative to RIP mutation content across the taxa examined (Figure 1; Supplementary Table S5). Although genome size was moderately correlated with RIP mutation content (Spearman's rank correlation coefficient $\left.r_{\mathrm{s}}=0.63, p<0.001\right)$, there were a number of instances of large genomes having very little RIP and small genomes rich in RIP mutations. For example, $1.5 \%$ of the 29 Megabases $(\mathrm{Mb})$ of the As. fumigatus genome is affected by RIP, while

TABLE 2 | A summary of genome-wide RIP statistics of representatives of each RIP class and control genome assemblies investigated in this study.

\begin{tabular}{|c|c|c|c|c|c|c|c|}
\hline \multirow{2}{*}{ Species } & \multirow{2}{*}{$\begin{array}{l}\text { Number of RIP- } \\
\text { positive windows }\end{array}$} & \multirow{2}{*}{ RIP class } & \multirow{2}{*}{$\begin{array}{c}\text { RIP-affected } \\
\text { genomic } \\
\text { proportion }(\%)^{2}\end{array}$} & \multicolumn{4}{|c|}{ Large RIP affected regions (LRARs) } \\
\hline & & & & $\begin{array}{c}\text { Number } \\
\text { detected }^{3}\end{array}$ & $\begin{array}{l}\text { Average size } \\
\text { (bp) }\end{array}$ & $\begin{array}{c}\text { Average GC } \\
(\%)\end{array}$ & $\begin{array}{c}\text { Combined size } \\
\text { (bp) }\end{array}$ \\
\hline Candida albicans & 0 & 1 & 0.0 & 0 & - & - & - \\
\hline Escherichia coli & 0 & 1 & 0.0 & 0 & - & - & - \\
\hline Encephalitozoon cuniculi & 1 & 1 & 0.0 & 0 & - & - & - \\
\hline Ceratocystis albifundus & 28 & 1 & 0.1 & 0 & - & - & - \\
\hline Fusarium graminearum & 206 & 2 & 0.3 & 2.0 & 6500.0 & 23.2 & 13,000 \\
\hline Fusarium poae & 4,472 & 3 & 4.8 & 182 & 10821.4 & 14.9 & $1,969,500$ \\
\hline Trichoderma reesei & 4,551 & 4 & 6.5 & 179 & 10662.0 & 15.6 & $1,908,500$ \\
\hline Verticillium dahlia & 5283 & 4 & 7.3 & 104 & 23966.4 & 25.1 & $2,492,500$ \\
\hline Leptosphaeria maculans & 10,225 & 5 & 11.8 & 275 & 7490.5 & 32.0 & $2,059,893$ \\
\hline Zymoseptoria tritici & 10,942 & 5 & 14.5 & 439 & 9670.0 & 15.2 & $4,245,131$ \\
\hline Neurospora crassa & 11,915 & 5 & 14.5 & 435 & 12347.1 & 28.4 & $5,371,000$ \\
\hline Pyrenophora teres & 32,740 & 6 & 29.5 & 406 & 38886.4 & 33.0 & $15,787,878$ \\
\hline
\end{tabular}

Organisms highlighted in bold served as control species. A full list of genome-wide RIP statistics is listed in Supplementary Table S5.

${ }^{1}$ Based on RIP index values.

${ }^{2}$ Proportion of the genome affected by RIP. Calculated using the total number of windows indicating RIP-positive index values against the total number of windows investigated for the entire genome sequence.

${ }^{3} L R A R$, large RIP affected genomic regions. More than 4,000 bp that are consecutively RIP affected. 
$0.55 \%$ of the $140 \mathrm{Mb}$ Bl. graminis f. sp. tritici genome constitute RIP mutations. RIP mutation content was also moderately correlated to repeat content estimated with REPET $\left(r_{\mathrm{s}}=0.52\right.$, $p<0.001$ ), but there were also numerous outliers. For example, only $0.65 \%$ of the Fusarium oxysporum genome with its high (23.5\%) repeat content constitutes RIP mutations, while the $N$. tetrasperma genome is characterized by a RIP mutation content of $10.3 \%$ and repeat content of only $0.5 \%$.

To simplify comparisons among the fungi examined, we recognized six classes of RIP (Figure 1). Fungi in Class 1 were those containing no evidence of RIP [i.e., no/few RIP-positive windows, where RIP constitute small/negligible proportions of the genome $(0$ to $<0.2 \%)$ ]. Class 6 fungi were those with genomes that are very highly affected by RIP (i.e., $>20 \%$ of the genome constituting RIP). The impact of RIP on the genomes of Classes 2-5 fungi were recorded as low $(>0.2$ to $<1 \%$ RIP), moderately low ( $>1$ to $<5 \%$ RIP), moderate $(>5$ to $<10 \%$ RIP), and moderately high ( $>10$ to $<20 \%$ RIP), respectively.

The 15 Class 1 genomes included all the Taphrinomycotina and Saccharomycotina investigated, as well as En. cuniculi. Overall, the RIP statistics generated for the Taphrinomycotina and Saccharomycotina were similar to those obtained for the negative control organisms (Supplementary Table S4). Among the Pezizomycotina, this was also observed for Monascus purpureus in the Eurotiomycetes and Ceratocystis albifundus in the Sordariomycetes. In these cases, no or only a few (1-70) RIP windows were detected, and these constituted very small proportions of the overall genome assemblies $(0-0.14 \%)$. Compared to the remainder of these genomes, the RIP-positive windows typically had low scoring RIP composite index values, and GC content was generally not reduced (Supplementary Table S5).

Twelve fungi were categorized as Class 2 as their genomes contained some LRARs (6-13) and low numbers of RIP-positive windows (180-798), constituting $0.37-0.65 \%$ of the individual genomes. These included members from the Xylonomycetes (Sy. buchneri and Xylona heveae), Sordariomycetes (Fusarium graminearum, Fusarium pseudograminearum, F. oxysporum, and Fusarium venenatum), Leotiomycetes (Botrytis cinerea), and Eurotiomycetes (Magnaporthe oryzae, Bl. graminis f. sp. tritici, and Aspergillus nidulans).

Of the 27 Classes 1 and 2 fungi, the genomes of six (i.e., Mo. purpureus, Sy. buchneri, Pi. kudriavzevii, Sa. complicata, Schizosaccharomyces pombe, and Ta. deformans) had low quality and were fragmented with lower BUSCO completeness scores. Five of these (i.e., Sy. buchneri, Pi. kudriavzevii, Sa. complicata, Sc. pombe, and Ta. deformans) were all from the Taphrinomycotina and Saccharomycotina in which RIP is also largely absent. However, in the case of Mo. purpureus from the Pezizomycotina, more research is needed to determine whether the low repeat and RIP mutation content estimated were artifacts of the quality of its genome.

The remaining 32 genomes all contained substantial evidence of RIP and belonged to RIP Classes 3-6. Sixteen of these were Class 3 and were recorded among the Orbiliomycetes, Pezizomycetes, Eurotiomycetes, Dothideomycetes, and Sordariomycetes (Figure 1).
RIP constituted between 1.03 and $4.81 \%$ of the genomes of these fungi and, except for Tuber borchii, and Tuber melanosporum, all had LRARs (17-183 per genome) with highly reduced GC contents and high scoring RIP composite index values (Supplementary Table S5).

Repeat-Induced Point Class 4 included eight fungi (i.e., Alternaria solani, Drechmeria coniospora, F. circinatum, Cladonia metacorallifera, Tr. reesei, Verticillium dahlia, Fusarium pininemorale, and Gyalolechia flavorubescens) from four taxonomic classes (Eurotiomycetes, Leotiomycetes, Dothideomycetes and Sordariomycetes) of the Pezizomycotina. These fungi had moderate levels of RIP (5 to 9\% RIP), and all of them had LRARs (81-182) that constituted a considerable proportion of the individual genomes ( 2.1 to $6.9 \%$ of the genome).

Lastly, seven genomes were placed in Class 5, while one was placed in Class 6. These genomes were from the Dothideomycetes (Z. tritici, and Le. maculans) and Sordariomycetes (N. tetrasperma, Valsa mali, N. crassa, Thermothelomyces thermophila, and Th. terrestris) and had high levels of RIP, which constituted more than $10 \%$ of the respective genome assemblies. Also, more than $10 \%$ of their genomes were predicted to be repetitive. RIP Class 6 was represented by $P y$. teres as its genome was highly affected by RIP (29.53\% RIP). The Classes 5 and 6 fungi all had many LRARs (289-406) that constituted large proportions (9.6-28.5\%) of the individual genome assemblies.

The results of chi-square tests of independence using a range of simulated data sets showed that our RIP categories were robust. In the case of the Class 2-6 genomes, these tests confirmed that the RIP distribution patterns obtained were not due to chance (Figure 1; Supplementary Table S5). For all the Class 2-6 genomes, the null expectation that the frequency of windows with RIP in a particular genome is the same as in the simulated data sets was rejected at a $99.99 \%$ confidence level $(p<0.01)$. As expected, this was not possible for any of the Class 1 genomes where the frequency of windows with RIP did not differ significantly from those recorded in the simulated data.

None of the Class 1 fungi encoded for a full complement of genes associated with the RIP pathway (Supplementary Table S3). The genomes of all Class 1 fungi, except Ce. albifundus, lacked a detectable DIM-7 gene, and most lacked one or both of the methyltransferases, RID and DIM-2. This was also the case for the Class 2 genomes of Bl. graminis f. sp. tritici (lacking HP-1, RID, DIM-2, and DIM-7) and Aspergillus oryzae (lacking DIM-2), as well as As. nidulans, Sy. buchneri, Bo. cinerea, and Sclerotinia sclerotiorum (all lacking DIM-7). The remaining Class 2 genomes contained both methyltransferases and the cofactors known to be involved in the DIM-2-mediated DNA methylation (i.e., F. oxysporum, F. graminearum, F. pseudograminearum, F. venenatum, Ma. oryzae, and X. heveae). The Class 3 genomes also contained all five DIM-2-associated cofactors, except for DIM-7 that was absent from Orbiliomycetes, Pezizomycetes, $\mathrm{Cl}$. metacorallifera, and Parastagonospora nodorum. Further, DIM-2 was absent from Ta. pinophilus, Pe. chrysogenum, Ce. manginecans, and Ce. fimbriata. Both Class 3 Orbiliomycetes Da. haptotyla, and Du. flagrans lacked DIM-2 and DIM-7. The Class 4-6 fungi contained all RIP-associated sequences 
investigated in this study. The only exception was As. fumigatus (Class 4), lacking DIM-2. The Class 5 Dothideomycetes species Z. tritici was notable as it lacked a complete copy of DIM-2 despite having a large proportion of its genome that constitutes RIP (Dhillon et al., 2010).

Based on our survey of the literature, the fungi in Classes 4-6 are capable of sexual reproduction (Wickerham et al., 1970; Sutton, 1982; Goto et al., 1987; Briza et al., 1990; Raju and Perkins, 1994; Gordon and Martyn, 1997; Keller et al., 1997; Britz et al., 1998; Harrington and Wingfield, 1998; Davis and Smith, 2001; Galagan et al., 2003; Dean et al., 2005; Rau et al., 2005; Rouxel and Balesdent, 2005; Paoletti et al., 2007; Rossi and Languasco, 2007; Tavanti et al., 2007; TeBeest et al., 2007; Bentley et al., 2008; Alby et al., 2009; Seidl et al., 2009; Mestre et al., 2010; Pereira et al., 2011; Rubini et al., 2011; Kurtzman and Robnett, 2012; Wada et al., 2012; Böhm et al., 2013; Studt et al., 2013; Short et al., 2014; Terhem and van Kan, 2014; Marin-Felix et al., 2015; Meng et al., 2015; Belfiori et al., 2016; Cowger et al., 2016; Doughan and Rollins, 2016; Gazis et al., 2016; Lee et al., 2016; Pelin et al., 2016; Son et al., 2016; Evans and Kirk, 2017; Manan, 2017; Randlane et al., 2017; Vaghefi et al., 2017; Vanheule et al., 2017; Yin et al., 2017; Yu et al., 2017; Carreté et al., 2018; Chi et al., 2019; Suffert et al., 2019; Wang et al., 2019a,b; Wu et al., 2020). Apart from a few instances where sexual stages have not been reported (indicated with " $U$ " in Figure 1), the only exception was the nematophagous fungus Dr. coniospora that apparently lack a sexual cycle completely (Zhang et al., 2016). In addition to sexual species, Classes 2 and 3 contained various fungi thought to be reproducing mainly asexually. The apparently asexual Class 3 fungi included the nematode-trapping species Du. flagrans (Wang et al., 2019b), the plant pathogen Colletotrichum higginsianum (O'Connell et al., 2012), and the mycoparasite Talaromyces pinophilus (Yilmaz et al., 2014). Class 2 fungi that are apparently asexual included the insect symbiont Sy. buchneri (Martinson, 2020), F. venenatum used in the production of mycoprotein (Leslie and Summerell, 2006), and the plant pathogen F. oxysporum (Braumann et al., 2008).

\section{DISCUSSION}

Genome-wide RIP data have allowed important insights on the taxonomic distribution of RIP in the Ascomycota. Our results provide evidence of RIP in most of the fungal sequences investigated. Extensive levels of RIP were detected in species such as Tr. reesei, N. crassa, Le. maculans, Th. terrestris, Py. teres, and Z. tritici. Fungi with extensive RIP often also were capable of sexual reproduction, had genomes rich in repetitive elements, and encoded the full complement of RIP-associated genes and genes associated with DNA methylation and RIP directed heterochromatic silencing. These findings were comparable to previous in silico studies on RIP (Clutterbuck, 2011; Meerupati et al., 2013; Testa et al., 2016; Li et al., 2017; van Wyk et al., 2019a). However, we present for the first-time evidence of RIP in F. pininemorale, $X$. haveae, and in representatives of the Lecanoromyces, G. flavorubescens and $\mathrm{Cl}$. metacorallifera.
Surprisingly, RIP was also recorded in a member of the Pezizomycetes, i.e., Tu. melanosporum, for which previous in silico studies (Clutterbuck, 2011; Chen et al., 2014; Hane et al., 2015) failed to detect RIP in its TE sequences. This may be because this fungus is no longer RIP-capable, and therefore RIP is absent from newly acquired TEs (Clutterbuck, 2011). The latter would be consistent with ancestral RIP activity in this species (Braumann et al., 2008), or the RIP-like mutations observed could have been the result of other mutational processes such as methylation induced premeiotically (MIP; Clutterbuck, 2011).

The patterns of genome-wide RIP observed for the Ascomycota support previous proposals regarding the evolutionary history of RIP. Initial emergence of RIP is thought to predate divergence of the Dikarya (Horns et al., 2012) between 500 and 650 million years ago (Lucking et al., 2009). However, we observed no evidence of RIP in the genomes of the Saccharomycotina and Taphrinomycotina, which is consistent with the notion that RIP capability was lost (Clutterbuck, 2011; Meerupati et al., 2013; Hane et al., 2015) in the ancestral lineages of these fungi following their divergence from the Pezizomycotina during the Paleozoic era (Prieto and Wedin, 2013). In comparison, RIP was particularly prominent among representatives of the Pezizomycotina, and we detected it in at least one representative of each taxonomic class in this subphylum. Additionally, RIP was recorded in all examined representatives of the two most basal groups of the Pezizomycotina, the Orbiliomycetes, and the Pezizomycetes (Spatafora et al., 2006). These data thus suggest that RIP is an ancient fungal genome defense strategy that has remained conserved for hundreds of millions of years across a broad taxonomic range.

Repeat-Induced Point mutations detected in apparently asexual fungi probably also represents signatures of ancestral RIP activity. Neither $F$. venenatum nor $F$. oxysporum have a known sexual stage (Leslie and Summerell, 2006; Braumann et al., 2008), but 0.86 and $0.65 \%$ of their respective genomes constituted RIP. This was also true for Dr. coniospora, in which $6.01 \%$ of its genome represented RIP, despite apparently lacking a sexual stage (Zhang et al., 2016). These findings thus suggest that RIP may be maintained for prolonged periods of time over numerous asexual generations. Alternatively, these fungi may have reproduced sexually in their evolutionary past or may maintain the ability to reproduce sexually but do so cryptically (Braumann et al., 2008). Similarly, the observed RIP in the $\mathrm{Tu}$. melanosporum genome most likely reflects ancestral RIP signatures that was acquired before loss of this process, as RIP is notably absent from newly acquired TEs and the genome also lacks LRARs (Clutterbuck, 2011; Chen et al., 2014; Hane et al., 2015). However, the possibility that RIP might be associated with processes other than sexual reproduction cannot be discounted. For example, recent studies on Dothideomycetes suggested the continued functioning of DIM-2 during mitotic cycles (Möller et al., 2020), but whether the DIM-2 activity is dependent on meiosis-linked signals or processes remains unclear.

Our results suggest that loss of RIP in the Saccharomycotina and Taphrinomycotina was likely associated with the loss of genes underlying the RIP pathway. Neither of these fungal groups encode for the full complement of the genes known 
to be involved in RIP, particularly the two methyltransferases, RID and DIM-2. Such losses probably also explain the lack of RIP capability in some of the more recently evolved lineages of the Pezizomycotina. For example, Bl. graminis f. sp. hordei is incapable of employing the RIP pathway as a genome defense mechanism due to the loss of key RIP genes (Spanu et al., 2010; Seidl and Thomma, 2017). This is consistent with the results of the current study, which showed that the genome of Bl. graminis f. sp. tritici, a taxon related to Bl. graminis $\mathrm{f}$. sp. hordei, lack RID and DIM-2, and displayed low levels of RIP $(0.55 \%$ of the genome constituted RIP; Class 2). RIP recorded in the $\mathrm{Bl}$. graminis $\mathrm{f}$. sp. tritici genome is most likely represented remnants of ancestral RIP (Braumann et al., 2008) that were acquired prior to the loss of RIP-associated genes.

Despite not maintaining all the genes underlying the RIP pathway, the genomes of some Ascomycota are rich in RIP mutations. A notable example is $Z$. tritici with its Class 5 genome characterized by extensive RIP in the absence of DIM-2-mediated DNA methylation (Dhillon et al., 2010). As previously speculated (Lorrain et al., 2020; Möller et al., 2020), such instances may reflect recent losses of DIM-2. However, studies on Metarhizium showed that RID and DIM-2 exert an additive effect on DNA methylation (Wang et al., 2017), and the functioning of these two pathways might thus influence RIP in a comparable fashion, where the loss of one of these pathways reduces RIP activity instead of completely abolishing it. Indeed, experimentally induced null mutations of either RID or DIM-2 resulted in reduced RIP activity in N. crassa (Freitag et al., 2002). Further, RID or DIM-2 mediated RIP may be individually dispensable (Gladyshev, 2017), allowing for occurrence of RIP in fungi where either one of the pathways are no longer maintained.

The extent of RIP differed extensively among the Ascomycota investigated. This was notable in the density of RIP that differed among closely related lineages, regardless of whether the fungi encoded similar sets of RIP-associated genes. For example, the Ce. albifundus genome contained no significant evidence of RIP, despite higher RIP levels being recorded in its close relatives $C e$. manginecans and Ce. fimbriata (Class 3). Another example is Fusarium where species differed vastly in the extent of RIP in their genomes, despite all containing a full complement of RIP-associated genes. For these fungi, three classes of RIP were recorded: Class 2 for F. oxysporum, F. pseudograminearum, F. graminearum, and F. venenatum, Class 3 for Fusarium poae and F. fujikuroi, and Class 4 for F. circinatum and F. pininemorale. The differences in the extent of RIP can be explained by differences in genomic content of TEs and repeats, reproductive strategies employed, complexities that arise during meiosis and mating, and/ or variation in expression levels of the RIP genes (Hane et al., 2015). In contrast, all representatives of the order Sordariales ( $N$. crassa, N. tertasperma, Thi. terrestris, and The. thermophila) had similar genome-wide RIP statistics, where a high level of RIP activity might represent a conserved and important heritable trait.

Our results suggested that TE integration into the host's genome and its subsequent response via RIP causes localized regions with biased sequence composition. For N. crassa, previous genome analyses revealed regional variation in RIP across the length of chromosomes, for which the total proportions of RIP can vary greatly (van Wyk et al., 2019b). Here, we complemented these data by showing that RIP is prominent in TE-rich regions of $N$. crassa and other fungi, as well as that centromeric and telomeric genomic regions are TE-rich with substantial levels of RIP. We also showed that RIP content generally coincided with regions of drastically reduced GC content, coupled with a higher frequency of TEs and repeated sequences. This is because TEs and other repeated sequences induce RIP (Cambareri et al., 1991), and extensive RIP directs localized GC-depletion, which is absent from the genomes of fungi that are not RIP-capable (Testa et al., 2016). The combined effects of TE integration and RIP likely also contribute to the formation of LRARs or long stretches of GC depleted genomic regions that are rich in RIP mutations. In fact, the occurrence of LRARs was recently shown to be a good indicator of extensive RIP (Lorrain et al., 2020). Similarly, extensive LRARs typically reflected high levels of RIP, and high repeat contents in the genomes investigated. Nevertheless, these data suggest that RIP directs biased sequence composition, maintained over large genomic regions, which constitutes considerable proportions of the overall genomes of many Ascomycota.

The combined effects of TE integration and RIP activity may also influence the genomic organization of Ascomycota (Frantzeskakis et al., 2019). One way in which these activities could exert their influence is in genome size. For example, fungi that have lost RIP more recently (e.g., Tu. melanosporum and species of Blumeria) are characterized by substantially enlarged genomes, which are the likely consequence of high levels of TE integration and uncontrolled transposition activity (Payen et al., 2016; Müller et al., 2019). In comparison, fungi from the Taphrinomycotina and Saccharomycotina that lost the ability to employ RIP much earlier presumably rely on other genome defense mechanisms such as MIP or meiotic silencing of unpaired DNA to maintain their more compact and smaller genomes (Gladyshev, 2017). Another way in which TEs and RIP activity can influence the genomic organization of Ascomycota is by driving or contributing to "genomic compartmentalization." Notable examples are Ve. dahlia and Le. maculans (Frantzeskakis et al., 2019) where combined TE and RIP activity led to the formation of a stable core compartment associated with house-keeping functions, and an accessory compartment that is more variable and highly dynamic (Frantzeskakis et al., 2019). By contrast, the genomes of most Pezizomycotina that no longer use RIP show less obvious genome compartmentalization (Dong et al., 2015; Frantzeskakis et al., 2019; Müller et al., 2019). Therefore, the availability of genome-wide RIP data may in future prove valuable to understanding such trends in genome evolution.

The data presented here suggest that the rigor of genomewide RIP analyses is dependent on the quality of the genome data being examined. Much of the recorded RIP was in the repeat-rich regions of genome assemblies. Many of these regions represent the telomeric, centromeric regions of chromosomes, and regions with a high frequency of TEs and repeat sequences. However, these genomic regions are often absent or fragmented from low quality genome assemblies (Testa et al., 2016). Accordingly, low-quality, low-coverage, and short read assemblies typically do not capture the genomic regions extensively affected 
by RIP (Testa et al., 2016). Therefore, care should be taken when interpreting genome-wide RIP statistics because low-quality genome assemblies may lead to an underestimation of the true extent of RIP on a genome-wide scale, although it can provide preliminary insight on the occurrence of RIP in the fungus in question.

The work presented in this study reports and summarizes the extent and occurrence of RIP by using a set of criteria to describe different Classes (1-6) of RIP. Although the use of these categories greatly simplified and streamlined our genome comparisons, separation of genomes based purely on the content of particular types of mutation may be misleading. However, as more information becomes available, the RIP classes delineated here may be revised and refined to improve their informativeness. For instance, for fungi with fragmented and/or incomplete genome assemblies, the true extent of RIP may remain underestimated until superior genome assemblies become available. Secondly, as each genome investigated in this study represents a single strain from a single population, the analysis of multiple isolates will allow for comparison of RIP classes within and across species. Thirdly, the exact molecular mechanism underlying RIP has not yet been fully determined, and conclusions drawn on the distribution of RIP-associated genes and the extent of RIP can thus be refined further as more genes are characterized and identified. Also, the effect size of each gene has not yet been quantitatively investigated, which may provide further insight on the molecular mechanisms underlying the occurrence and extent of RIP in fungi. For instance, some representatives of the Taphrinomycotina have detectable homologs for many of the genes associated with RIP, yet evidence of RIP in these fungi are lacking. Therefore, functional investigations such as expression analyses and functional gene knockouts might provide greater insight on the RIP capability of the fungi investigated in this study.

In conclusion, genome-wide RIP data for representatives of the Ascomycota have provided a comprehensive view of the occurrence, extent, and distribution of RIP. Data generated using a sliding window-based method yielded superior results compared to those generated using TE sequence alignments. Also, application of these sliding window-based analyses, where all parameters were considered within the same genetic context, reduced the occurrence of erroneous identification of RIP. It enabled the evaluation of the extent and occurrence of RIP for each sequenced representative investigated using a uniform approach. The results of this study have further provided the first reported evidence of RIP in some fungi and for sequenced representatives displaying previously unknown traces of ancestral RIP. Additionally, this work provided information on RIP from fungi representing varying degrees of divergence from the ancestor of the

\section{REFERENCES}

Alby, K., Schaefer, D., and Bennett, R. J. (2009). Homothallic and heterothallic mating in the opportunistic pathogen Candida albicans. Nature 460, 890-893. doi: 10.1038/nature08252

Belfiori, B., Riccioni, C., Paolocci, F., and Rubini, A. (2016). Characterization of the reproductive mode and life cycle of the whitish truffle T. borchii. Mycorrhiza 26, 515-527. doi: 10.1007/s00572-016-0689-0
Ascomycota, thus allowing for conclusions to be drawn regarding the taxonomic range and evolutionary history of RIP. Previous work lacked a uniform method to described observed variation of RIP between different species. Here, we presented six categories to summarize and describe the variation in extent of RIP observed for these fungi. The body of knowledge generated in this study forms a baseline to enable future comparative analyses of RIP of newly sequenced genomes. Moreover, the data generated here can serve as a basis for functional investigations to address questions relating to the biological and molecular determinants directing the observed variation in RIP of the Ascomycota.

\section{DATA AVAILABILITY STATEMENT}

The original contributions presented in the study are included in the article/Supplementary Material, further inquiries can be directed to the corresponding author.

\section{AUTHOR CONTRIBUTIONS}

SW, ES, LV, NM, and BW: conceptualization, methodology, writing - review, and editing. SW, ES, BW, and NM: software. SW, ES, and LV: validation and investigation. SW and LV: formal analysis. ES, LV, and BW: resources. SW, ES, LV, and BW: data curation. SW and ES: writing - original draft preparation, critical revisions, and writing. SW: visualization. ES, LV, NM, and BW: supervision. ES: project administration. BW and ES: funding acquisition. All authors contributed to the article and approved the submitted version.

\section{FUNDING}

This research was funded by the University of Pretoria, the Department of Science and Innovation (DSI) and the National Research Foundation (NRF) via the Centre of Excellence in Plant Health Biotechnology and the South African Research Chairs Initiative (SARChI) Chair in Fungal Genomics. The APC was funded by the University of Pretoria.

\section{SUPPLEMENTARY MATERIAL}

The Supplementary Material for this article can be found online at: https://www.frontiersin.org/articles/10.3389/fmicb.2020.622368/ full\#supplementary-material

Bentley, A. R., Summerell, B. A., and Burgess, L. W. (2008). Sexual compatibility in Fusarium pseudograminearum (Gibberella coronicola). Mycol. Res. 112, 1101-1106. doi: 10.1016/j.mycres.2008.04.016

Bewick, A. J., Hofmeister, B. T., Powers, R. A., Mondo, S. J., Grigoriev, I. V., James, T. Y., et al. (2019). Diversity of cytosine methylation across the fungal tree of life. Nat. Ecol. Evol. 3, 479-490. doi: 10.1038/s41559-019-0810-9

Billiard, S., López-Villavicencio, M., Hood, M., and Giraud, T. (2012). Sex, outcrossing and mating types: unsolved questions in fungi 
and beyond. J. Evol. Biol. 25, 1020-1038. doi: 10.1111/j.1420-9101. 2012.02495.x

Böhm, J., Hoff, B., O’Gorman, C. M., Wolfers, S., Klix, V., Binger, D., et al. (2013). Sexual reproduction and mating-type-mediated strain development in the penicillin-producing fungus Penicillium chrysogenum. Proc. Natl. Acad. Sci. 110, 1476-1481. doi: 10.1073/pnas.1217943110

Bowman, B. H., Taylor, J. W., and White, T. J. (1992). Molecular evolution of the fungi: human pathogens. Mol. Biol. Evol. 9, 893-904. doi: 10.1093/ oxfordjournals.molbev.a040766

Braumann, I., Van Den Berg, M., and Kempken, F. (2008). Repeat induced point mutation in two asexual fungi, Aspergillus niger and Penicillium chrysogenum. Curr. Genet. 53, 287-297. doi: 10.1007/s00294-008-0185-y

Britz, H., Wingfield, M., Coutinho, T., Marasas, W., and Leslie, J. (1998). Female fertility and mating type distribution in a south African population of Fusarium subglutinans fsp. pini. Appl. Environ. Microbiol. 64, 2094-2095. doi: 10.1128/AEM.64.6.2094-2095.1998

Briza, P., Breitenbach, M., Ellinger, A., and Segall, J. (1990). Isolation of two developmentally regulated genes involved in spore wall maturation in Saccharomyces cerevisiae. Genes Dev. 4, 1775-1789. doi: 10.1101/gad.4.10.1775

Cambareri, E., Singer, M., and Selker, E. (1991). Recurrence of repeat-induced point mutation (RIP) in Neurospora crassa. Genetics 127, 699-710.

Carreté, L., Ksiezopolska, E., Pegueroles, C., Gómez-Molero, E., Saus, E., Iraola-Guzmán, S., et al. (2018). Patterns of genomic variation in the opportunistic pathogen Candida glabrata suggest the existence of mating and a secondary association with humans. Curr. Biol. 28, 15.e7-27.e7. doi: 10.1016/j.cub.2017.11.027

Chen, P. -Y., Montanini, B., Liao, W. -W., Morselli, M., Jaroszewicz, A., Lopez, D., et al. (2014). A comprehensive resource of genomic, epigenomic and transcriptomic sequencing data for the black truffle Tuber melanosporum. GigaScience 3:25. doi: 10.1186/2047-217X-3-25

Chi, N., Thu, P., Hinh, T., and Dell, B. (2019). Management of Ceratocystis manginecans in plantations of Acacia through optimal pruning and site selection. Australas. Plant Pathol. 48, 343-350. doi: 10.1007/s13313-019-00635-1

Clutterbuck, A. J. (2011). Genomic evidence of repeat-induced point mutation (RIP) in filamentous ascomycetes. Fungal Genet. Biol. 48, 306-326. doi: 10.1016/j.fgb.2010.09.002

Cowger, C., Parks, R., and Kosman, E. (2016). Structure and migration in US Blumeria graminis f. sp. tritici populations. Phytopathology 106, 295-304. doi: 10.1094/PHYTO-03-15-0066-R

Davis, L., and Smith, G. R. (2001). Meiotic recombination and chromosome segregation in Schizosaccharomyces pombe. Proc. Natl. Acad. Sci. 98, 8395-8402. doi: 10.1073/pnas. 121005598

de Oliveira, J. M. P. F., and de Graaff, L. H. (2011). Proteomics of industrial fungi: trends and insights for biotechnology. Appl. Microbiol. Biotechnol. 89, 225-237. doi: 10.1007/s00253-010-2900-0

Dean, R. A., Talbot, N. J., Ebbole, D. J., Farman, M. L., Mitchell, T. K., Orbach, M. J., et al. (2005). The genome sequence of the rice blast fungus Magnaporthe grisea. Nature 434, 980-986. doi: 10.1038/nature03449

Dhillon, B., Cavaletto, J. R., Wood, K. V., and Goodwin, S. B. (2010). Accidental amplification and inactivation of a methyltransferase gene eliminates cytosine methylation in Mycosphaerella graminicola. Genetics 186, 67-77. doi: 10.1534/ genetics.110.117408

Dong, S., Raffaele, S., and Kamoun, S. (2015). The two-speed genomes of filamentous pathogens: waltz with plants. Curr. Opin. Genet. Dev. 35, 57-65. doi: 10.1016/j.gde.2015.09.001

Doughan, B., and Rollins, J. A. (2016). Characterization of MAT gene functions in the life cycle of Sclerotinia sclerotiorum reveals a lineage-specific MAT gene functioning in apothecium morphogenesis. Fungal Biol. 120, 1105-1117. doi: 10.1016/j.funbio.2016.06.007

Douglass, A. P., Offei, B., Braun-Galleani, S., Coughlan, A. Y., Martos, A. A., Ortiz-Merino, R. A., et al. (2018). Population genomics shows no distinction between pathogenic Candida krusei and environmental Pichia kudriavzevii: one speciesfour names. PLoS Pathog. 14:e1007138. doi: 10.1371/journal.ppat.1007138

Ellison, C. E., Stajich, J. E., Jacobson, D. J., Natvig, D. O., Lapidus, A., Foster, B., et al. (2011). Massive changes in genome architecture accompany the transition to self-fertility in the filamentous fungus Neurospora tetrasperma. Genetics 189, 55-69. doi: 10.1534/genetics.111.130690

Evans, H. C., and Kirk, P. M. (2017). "Systematics of Pochonia" in Perspectives in sustainable nematode management through Pochonia chlamydosporia applications for root and rhizosphere health. eds. R. H. Manzanilla-López and L. V. Lopez-Llorca (Cham: Springer), 21-43.

Flutre, T., Duprat, E., Feuillet, C., and Quesneville, H. (2011). Considering transposable element diversification in de novo annotation approaches. PLoS One 6:e16526. doi: 10.1371/journal.pone.0016526

Frantzeskakis, L., Kusch, S., and Panstruga, R. (2019). The need for speed: compartmentalized genome evolution in filamentous phytopathogens. Mol. Plant Pathol. 20, 3-7. doi: 10.1111/mpp.12738

Freitag, M., Williams, R. L., Kothe, G. O., and Selker, E. U. (2002). A cytosine methyltransferase homologue is essential for repeat-induced point mutation in Neurospora crassa. Proc. Natl. Acad. Sci. 99, 8802-8807. doi: 10.1073/ pnas.132212899

Fudal, I., Ross, S., Brun, H., Besnard, A. -L., Ermel, M., Kuhn, M. -L., et al. (2009). Repeat-induced point mutation (RIP) as an alternative mechanism of evolution toward virulence in Leptosphaeria maculans. Mol. Plant-Microbe Interact. 22, 932-941. doi: 10.1094/MPMI-22-8-0932

Galagan, J. E., Calvo, S. E., Borkovich, K. A., Selker, E. U., Read, N. D., Jaffe, D., et al. (2003). The genome sequence of the filamentous fungus Neurospora crassa. Nature 422, 859-868. doi: 10.1038/nature01554

Gazis, R., Kuo, A., Riley, R., LaButti, K., Lipzen, A., Lin, J., et al. (2016). The genome of Xylona heveae provides a window into fungal endophytism. Fungal Biol. 120, 26-42. doi: 10.1016/j.funbio.2015.10.002

Gessaman, J. D., and Selker, E. U. (2017). Induction of H3K9me3 and DNA methylation by tethered heterochromatin factors in Neurospora crassa. Proc. Natl. Acad. Sci. 114, E9598-E9607. doi: 10.1073/pnas.1715049114

Gladyshev, E. (2017). "Repeat-induced point mutation and other genome defense mechanisms in fungi” in The fungal kingdom. eds. J. Heitman, B. J. Howlett, P. W. Crous, E. H. Stukenbrock, T. Y. James and N. A. R. Gow (Washington, DC: ASM Press), 687-699.

Gordon, T., and Martyn, R. (1997). The evolutionary biology of Fusarium oxysporum. Annu. Rev. Phytopathol. 35, 111-128. doi: 10.1146/annurev. phyto.35.1.111

Goto, S., Sugiyama, J., Hamamoto, M., and Komagata, K. (1987). Saitoella, a new anamorph genus in the Cryptococcaceae to accommodate two Himalayan yeast isolates formerly identified as Rhodotorula glutinis. J. Gen. Appl. Microbiol. $33,75-85$.

Grigoriev, I. V., Cullen, D., Goodwin, S. B., Hibbett, D., Jeffries, T. W., Kubicek, C. P., et al. (2011). Fueling the future with fungal genomics. Mycology 2, 192-209. doi: 10.1080/21501203.2011.584577

Hane, J. K., and Oliver, R. P. (2008). RIPCAL: a tool for alignment-based analysis of repeat-induced point mutations in fungal genomic sequences. BMC Bioinformatics 9:478. doi: 10.1186/1471-2105-9-478

Hane, J. K., and Oliver, R. P. (2010). In silico reversal of repeat-induced point mutation (RIP) identifies the origins of repeat families and uncovers obscured duplicated genes. BMC Genomics 11:655. doi: 10.1186/14712164-11-655

Hane, J. K., Williams, A. H., Taranto, A. P., Solomon, P. S., and Oliver, R. P. (2015). "Repeat-induced point mutation: a fungal-specific, endogenous mutagenesis process" in Genetic transformation Systems in Fungi. Vol. 2. eds. M. A. van den Berg and K. Maruthachalam (Switzerland: Springer International Publishing), 55-68.

Harrington, T. C., and Wingfield, M. J. (1998). The Ceratocystis species on conifers. Can. J. Bot. 76, 1446-1457.

He, C., Zhang, Z., Li, B., and Tian, S. (2020). The pattern and function of DNA methylation in fungal plant pathogens. Microorganisms 8:227. doi: $10.3390 /$ microorganisms 8020227

Horns, F., Petit, E., Yockteng, R., and Hood, M. E. (2012). Patterns of repeatinduced point mutation in transposable elements of basidiomycete fungi. Genome Biol. Evol. 4, 240-247. doi: 10.1093/gbe/evs005

Irelan, J. T., Hagemann, A. T., and Selker, E. U. (1994). High frequency repeatinduced point mutation (RIP) is not associated with efficient recombination in Neurospora. Genetics 138, 1093-1103.

Katoh, K., Rozewicki, J., and Yamada, K. D. (2019). MAFFT online service: multiple sequence alignment, interactive sequence choice and visualization. Brief. Bioinform. 20, 1160-1166. doi: 10.1093/bib/bbx108

Keller, S. M., McDermott, J. M., Pettway, R., Wolfe, M., and McDonald, B. (1997). Gene flow and sexual reproduction in the wheat glume blotch pathogen Phaeosphaeria nodorum (anamorph Stagonospora nodorum). Phytopathology 87, 353-358. 
King, R., Urban, M., Hammond-Kosack, M. C., Hassani-Pak, K., and Hammond-Kosack, K. E. (2015). The completed genome sequence of the pathogenic ascomycete fungus Fusarium graminearum. BMC Genomics 16:544. doi: 10.1186/s12864-015-1756-1

Kumar, P., Chatli, M., Mehta, N., Singh, P., Malav, O., and Verma, A. K. (2017). Meat analogues: health promising sustainable meat substitutes. Crit. Rev. Food Sci. Nutr. 57, 923-932. doi: 10.1080/10408398.2014.939739

Kurtzman, C. P., and Robnett, C. J. (2012). Saitoellacoloradoensis sp. nov., a new species of the Ascomycota, subphylum Taphrinomycotina. Antonie Van Leeuwenhoek 101, 795-802. doi: 10.1007/s10482-011-9694-7

Lee, D. -H., Roux, J., Wingfield, B. D., Barnes, I., Mostert, L., and Wingfield, M. J. (2016). The genetic landscape of Ceratocystis albifundus populations in South Africa reveals a recent fungal introduction event. Fungal Biol. 120, 690-700. doi: 10.1016/j.funbio.2016.03.001

Leslie, J., and Summerell, B. (2006). Fusarium laboratory workshops-a recent history. Mycotoxin Res. 22:73. doi: 10.1007/BF02956766

Letunic, I., and Bork, P. (2016). Interactive tree of life (iTOL) v3: an online tool for the display and annotation of phylogenetic and other trees. Nucleic Acids Res. 44, W242-W245. doi: 10.1093/nar/gkw290

Lewis, Z. A., Adhvaryu, K. K., Honda, S., Shiver, A. L., Knip, M., Sack, R., et al. (2010). DNA methylation and normal chromosome behavior in Neurospora depend on five components of a histone methyltransferase complex DCDC. PLoS Genet. 6:e1001196. doi: 10.1371/journal.pgen.1001196

Lewis, Z. A., Honda, S., Khlafallah, T. K., Jeffress, J. K., Freitag, M., Mohn, F., et al. (2009). Relics of repeat-induced point mutation direct heterochromatin formation in Neurospora crassa. Genome Res. 19, 427-437. doi: 10.1101/ gr.086231.108

Li, W. -C., Huang, C. -H., Chen, C. -L., Chuang, Y. -C., Tung, S. -Y., and Wang, T. -F. (2017). Trichoderma reesei complete genome sequence, repeatinduced point mutation, and partitioning of CAZyme gene clusters. Biotechnol. Biofuels 10:170. doi: 10.1186/s13068-017-0825-x

Lorrain, C., Feurtey, A., Moller, M., Haueisen, J., and Stukenbrock, E. H. (2020). "Dynamics of transposable elements in recently diverged fungal pathogens: lineage-specific transposable element content and efficiency of genome defences." bioRxiv [Preprint]. doi: 10.1101/2020.05.13.092635

Lucking, R., Huhndorf, S., Pfister, D. H., Plata, E. R., and Lumbsch, H. T. (2009). Fungi evolved right on track. Mycologia 101, 810-822. doi: 10.3852/09-016

Manan, M. (2017). The morphology and structure of red pigment producing fungus: Monascus purpureus. J. Microbiol. Exp. 5:00138. doi: 10.15406/ jmen.2017.05.00138

Margolin, B. S., Garrett-Engele, P. W., Stevens, J. N., Fritz, D. Y., Garrett-Engele, C., Metzenberg, R. L., et al. (1998). A methylated Neurospora 5S rRNA pseudogene contains a transposable element inactivated by repeat-induced point mutation. Genetics 149, 1787-1797

Marin-Felix, Y., Stchigel, A. M., Miller, A. N., Guarro, J., and Cano-Lira, J. F. (2015). A re-evaluation of the genus Myceliophthora (Sordariales, Ascomycota): its segregation into four genera and description of Corynascus fumimontanus sp. nov. Mycologia 107, 619-632. doi: 10.3852/14-228

Martens, E., and Demain, A. L. (2017). "An overview of the industrial aspects of antibiotic discovery" in Microbial resources. ed. I. Kurtböke (London, United Kingdom: Elsevier Academic Press), 149-168.

Martinson, V. G. (2020). Rediscovering a forgotten system of symbiosis: historical perspective and future potential. Gene 11:1063. doi: 10.3390/genes11091063

McLaughlin, D. J., Hibbett, D. S., Lutzoni, F., Spatafora, J. W., and Vilgalys, R. (2009). The search for the fungal tree of life. Trends Microbiol. 17, 488-497. doi: 10.1016/j.tim.2009.08.001

Meerupati, T., Andersson, K. -M., Friman, E., Kumar, D., Tunlid, A., and Ahrén, D. (2013). Genomic mechanisms accounting for the adaptation to parasitism in nematode-trapping fungi. PLoS Genet. 9:e1003909. doi: 10.1371/ journal.pgen.1003909

Meng, J. -W., Zhu, W., He, M. -H., Wu, E. -J., Duan, G. -H., Xie, Y. -K., et al. (2015). Population genetic analysis reveals cryptic sex in the phytopathogenic fungus Alternaria alternata. Sci. Rep. 5:18250. doi: 10.1038/srep 18250

Mestre, M., Ulloa, J., Rosa, C., Lachance, M., and Fontenla, S. (2010). Lachancea nothofagi sp. nov., a yeast associated with Nothofagus species in Patagonia, Argentina. Int. J. Syst. Evol. Microbiol. 60, 2247-2250. doi: 10.1099/ijs.0.018929-0

Möller, M., Habig, M., Lorrain, C., Feurtey, A., Haueisen, J., Fagundes, W. C., et al. (2020). "Recent loss of the Dim2 cytosine DNA methyltransferase impacts mutation rate and evolution in a fungal plant pathogen." bioRxiv [Preprint]. doi: 10.1101/2020.03.27.012203

Mueller, G. M., and Schmit, J. P. (2007). Fungal biodiversity: what do we know? What can we predict? Biodivers. Conserv. 16, 1-5. doi: 10.1007/s10531006-9117-7

Müller, M. C., Praz, C. R., Sotiropoulos, A. G., Menardo, F., Kunz, L., Schudel, S., et al. (2019). A chromosome-scale genome assembly reveals a highly dynamic effector repertoire of wheat powdery mildew. New Phytol. 221, 2176-2189. doi: $10.1111 / \mathrm{nph} .15529$

O'Connell, R. J., Thon, M. R., Hacquard, S., Amyotte, S. G., Kleemann, J., Torres, M. F., et al. (2012). Lifestyle transitions in plant pathogenic Colletotrichum fungi deciphered by genome and transcriptome analyses. Nat. Genet. 44, 1060-1065. doi: 10.1038/ng.2372

Paoletti, M., Seymour, F. A., Alcocer, M. J., Kaur, N., Calvo, A. M., Archer, D. B., et al. (2007). Mating type and the genetic basis of self-fertility in the model fungus Aspergillus nidulans. Curr. Biol. 17, 1384-1389. doi: 10.1016/j. cub.2007.07.012

Payen, T., Murat, C., and Martin, F. (2016). Reconstructing the evolutionary history of gypsy retrotransposons in the Perigord black truffle (Tuber melanosporum Vittad.). Mycorrhiza 26, 553-563. doi: 10.1007/s00572-0160692-5

Payne, C. M., Knott, B. C., Mayes, H. B., Hansson, H., Himmel, M. E., Sandgren, M., et al. (2015). Fungal cellulases. Chem. Rev. 115, 1308-1448. doi: $10.1021 / \mathrm{cr} 500351 \mathrm{c}$

Pelin, A., Moteshareie, H., Sak, B., Selman, M., Naor, A., Eyahpaise, M. -È., et al. (2016). The genome of an Encephalitozoon cuniculi type III strain reveals insights into the genetic diversity and mode of reproduction of a ubiquitous vertebrate pathogen. Heredity 116, 458-465. doi: 10.1038/hdy.2016.4

Pereira, L. F., Costa, C. R. L. Jr., Brasileiro, B. T. R. V., and de Morais, M. A. Jr. (2011). Lachancea mirantina sp. nov., an ascomycetous yeast isolated from the cachaca fermentation process. Int. J. Syst. Evol. Microbiol. 61, 989-992. doi: $10.1099 /$ ijs.0.020008-0

Prieto, M., and Wedin, M. (2013). Dating the diversification of the major lineages of Ascomycota (Fungi). PLoS One 8:e65576. doi: 10.1371/journal. pone.0065576

Quesneville, H., Bergman, C. M., Andrieu, O., Autard, D., Nouaud, D., Ashburner, M., et al. (2005). Combined evidence annotation of transposable elements in genome sequences. PLoS Comput. Biol. 1:e22. doi: 10.1371/ journal.pcbi.0010022

Raju, N. B., and Perkins, D. D. (1994). Diverse programs of ascus development in pseudohomothallic species of Neurospora, Gelasinospora and Podospora. Dev. Genet. 15, 104-118. doi: 10.1002/dvg.1020150111

Randlane, T., Tullus, T., Saag, A., Lutter, R., Tullus, A., Helm, A., et al. (2017). Diversity of lichens and bryophytes in hybrid aspen plantations in Estonia depends on landscape structure. Can. J. For. Res. 47, 1202-1214. doi: 10.1139/ cjfr-2017-0080

Rau, D., Maier, F. J., Papa, R., Brown, A. H., Balmas, V., Saba, E., et al. (2005). Isolation and characterization of the mating-type locus of the barley pathogen Pyrenophora teres and frequencies of mating-type idiomorphs within and among fungal populations collected from barley landraces. Genome 48, 855-869. doi: 10.1139/g05-046

Rossi, V., and Languasco, L. (2007). Influence of environmental conditions on spore production and budding in Taphrina deformans, the causal agent of peach leaf curl. Phytopathology 97, 359-365. doi: 10.1094/PHYTO-97-3-0359

Rouxel, T., and Balesdent, M. -H. (2005). The stem canker (blackleg) fungus, Leptosphaeria maculans, enters the genomic era. Mol. Plant Pathol. 6, 225-241. doi: 10.1111/j.1364-3703.2005.00282.x

Rouxel, T., Grandaubert, J., Hane, J. K., Hoede, C., van de Wouw, A. P., Couloux, A., et al. (2011). Effector diversification within compartments of the Leptosphaeria maculans genome affected by repeat-induced point mutations. Nat. Commun. 2:202. doi: 10.1038/ncomms1189

Rubini, A., Belfiori, B., Riccioni, C., Tisserant, E., Arcioni, S., Martin, F., et al. (2011). Isolation and characterization of MAT genes in the symbiotic ascomycete Tuber melanosporum. New Phytol. 189, 710-722. doi: 10.1111/j. 1469-8137.2010.03492.x

Schoch, C. L., Sung, G. -H., López-Giráldez, F., Townsend, J. P., Miadlikowska, J., Hofstetter, V., et al. (2009). The Ascomycota tree of life: a phylum-wide phylogeny clarifies the origin and evolution of fundamental reproductive and ecological traits. Syst. Biol. 58, 224-239. doi: 10.1093/sysbio/syp020 
Seidl, V., Seibel, C., Kubicek, C. P., and Schmoll, M. (2009). Sexual development in the industrial workhorse Trichoderma reesei. Proc. Natl. Acad. Sci. 106, 13909-13914. doi: 10.1073/pnas.0904936106

Seidl, M. F., and Thomma, B. P. (2017). Transposable elements direct the coevolution between plants and microbes. Trends Genet. 33, 842-851. doi: 10.1016/j.tig.2017.07.003

Selker, E. U. (1990). Premeiotic instability of repeated sequences in Neurospora crassa. Annu. Rev. Genet. 24, 579-613. doi: 10.1146/annurev.ge.24.120190.003051

Selker, E. U. (2002). Repeat-induced gene silencing in fungi. Adv. Genet. 46, 439-450. doi: 10.1016/s0065-2660(02)46016-6

Selker, E. U., and Garrett, P. W. (1988). DNA sequence duplications trigger gene inactivation in Neurospora crassa. Proc. Natl. Acad. Sci. 85, 6870-6874. doi: $10.1073 /$ pnas.85.18.6870

Selker, E. U., Tountas, N. A., Cross, S. H., Margolin, B. S., Murphy, J. G., Bird, A. P., et al. (2003). The methylated component of the Neurospora crassa genome. Nature 422, 893-897. doi: 10.1038/nature01564

Short, D. P., Gurung, S., Hu, X., Inderbitzin, P., and Subbarao, K. V. (2014). Maintenance of sex-related genes and the co-occurrence of both mating types in Verticillium dahliae. PLoS One 9:e112145. doi: 10.1371/journal. pone. 0112145

Simão, F. A., Waterhouse, R. M., Ioannidis, P., Kriventseva, E. V., and Zdobnov, E. M. (2015). BUSCO: assessing genome assembly and annotation completeness with single-copy orthologs. Bioinformatics 31, 3210-3212. doi: 10.1093/ bioinformatics/btv351

Son, H., Lim, J. Y., Lee, Y., and Lee, Y. -W. (2016). Utilization of a conidiadeficient mutant to study sexual development in Fusarium graminearum. PLoS One 11:e0155671. doi: 10.1371/journal.pone.0155671

Spanu, P. D., Abbott, J. C., Amselem, J., Burgis, T. A., Soanes, D. M., Stüber, K., et al. (2010). Genome expansion and gene loss in powdery mildew fungi reveal tradeoffs in extreme parasitism. Science 330, 1543-1546. doi: 10.1126/ science. 1194573

Spatafora, J. W., Sung, G. -H., Johnson, D., Hesse, C., O’Rourke, B., Serdani, M., et al. (2006). A five-gene phylogeny of Pezizomycotina. Mycologia 98, 1018-1028. doi: 10.3852/mycologia.98.6.1018

Stothard, P. (2000). The sequence manipulation suite: JavaScript programs for analyzing and formatting protein and DNA sequences. BioTechniques 28, 1102-1104. doi: 10.2144/00286ir01

Studt, L., Humpf, H. -U., and Tudzynski, B. (2013). Signaling governed by G proteins and cAMP is crucial for growth, secondary metabolism and sexual development in Fusarium fujikuroi. PLoS One 8:e58185. doi: 10.1371/journal. pone.0058185

Suffert, F., Delestre, G., and Gélisse, S. (2019). Sexual reproduction in the fungal foliar pathogen Zymoseptoria tritici is driven by antagonistic density dependence mechanisms. Microb. Ecol. 77, 110-123. doi: 10.1007/ s00248-018-1211-3

Sun, Y., Svedberg, J., Hiltunen, M., Corcoran, P., and Johannesson, H. (2017). Large-scale suppression of recombination predates genomic rearrangements in Neurospora tetrasperma. Nat. Commun. 8:1140. doi: 10.1038/s41467-01701317-6

Sutton, J. (1982). Epidemiology of wheat head blight and maize ear rot caused by Fusarium graminearum. Can. J. Plant Pathol. 4, 195-209.

Tavanti, A., Hensgens, L. A., Ghelardi, E., Campa, M., and Senesi, S. (2007). Genotyping of Candida orthopsilosis clinical isolates by amplification fragment length polymorphism reveals genetic diversity among independent isolates and strain maintenance within patients. J. Clin. Microbiol. 45, 1455-1462. doi: 10.1128/JCM.00243-07

TeBeest, D. O., Guerber, C., and Ditmore, M. (2007). "Symptoms and signs" in The plant health instructor. ed. C. Little (St. Paul, MN, USA: The American Phytopathological Society).

Terhem, R. B., and van Kan, J. A. (2014). Functional analysis of hydrophobin genes in sexual development of Botrytis cinerea. Fungal Genet. Biol. 71, 42-51. doi: 10.1016/j.fgb.2014.08.002

Testa, A. C., Oliver, R. P., and Hane, J. K. (2016). OcculterCut: a comprehensive survey of AT-rich regions in fungal genomes. Genome Biol. Evol. 8, 2044-2064. doi: 10.1093/gbe/evw121

Vaghefi, N., Kikkert, J. R., Bolton, M. D., Hanson, L. E., Secor, G. A., and Pethybridge, S. J. (2017). De novo genome assembly of Cercospora beticola for microsatellite marker development and validation. Fungal Ecol. 26, 125-134. doi: 10.1016/j.funeco.2017.01.006
Van de Wouw, A. P., Elliott, C. E., Popa, K. M., and Idnurm, A. (2018). Analysis of repeat induced point (RIP) mutations in Leptosphaeria maculans indicates variability in the RIP process between fungal species. Genetics 2018:301712. doi: 10.1534/genetics.118.301712

van Wyk, S., Harrison, C. H., Wingfield, B. D., De Vos, L., van der Merwe, N. A., and Steenkamp, E. T. (2019b). The RIPper, a web-based tool for genomewide quantification of repeat-induced point (RIP) mutations. PeerJ 7:e7447. doi: $10.7717 /$ peerj.7447

van Wyk, S., Wingfield, B. D., De Vos, L., Santana, Q. C., Van der Merwe, N. A., and Steenkamp, E. T. (2018). Multiple independent origins for a subtelomeric locus associated with growth rate in Fusarium circinatum. IMA fungus 9:27. doi: 10.5598/imafungus.2018.09.01.03

van Wyk, S., Wingfield, B. D., De Vos, L., van der Merwe, N. A., Santana, Q. C., and Steenkamp, E. T. (2019a). Repeat-induced point mutations drive divergence between Fusarium circinatum and its close relatives. Pathogens 8:298. doi: $10.3390 /$ pathogens 8040298

Vanheule, A., De Boevre, M., Moretti, A., Scauflaire, J., Munaut, F., De Saeger, S., et al. (2017). Genetic divergence and chemotype diversity in the Fusarium head blight pathogen Fusarium poae. Toxins 9:255. doi: 10.3390/toxins9090255

Wada, R., Maruyama, J. -I., Yamaguchi, H., Yamamoto, N., Wagu, Y., Paoletti, M., et al. (2012). Presence and functionality of mating type genes in the supposedly asexual filamentous fungus Aspergillus oryzae. Appl. Environ. Microbiol. 78, 2819-2829. doi: 10.1128/AEM.07034-11

Wang, X., Bai, F., Bensch, K., Meijer, M., Sun, B., Han, Y., et al. (2019a). Phylogenetic re-evaluation of Thielavia with the introduction of a new family Podosporaceae. Stud. Mycol. 93, 155-252. doi: 10.1016/j.simyco.2019.08.002

Wang, Y., Wang, T., Qiao, L., Zhu, J., Fan, J., Zhang, T., et al. (2017). DNA methyltransferases contribute to the fungal development, stress tolerance and virulence of the entomopathogenic fungus Metarhizium robertsii. Appl. Microbiol. Biotechnol. 101, 4215-4226. doi: 10.1007/s00253-017-8197-5

Wang, B., Zhang, N., Gong, P. -T., Li, J., Yang, J., Zhang, X., et al. (2019b). Effect of temperature, $\mathrm{pH}$, physical and chemical factors on germination rate of the chlamydospores of the nematophagous fungus Duddingtonia flagrans. FEMS Microbiol. Lett. 366:fnz212. doi: 10.1093/femsle/fnz212

Waterhouse, R. M., Seppey, M., Simão, F. A., Manni, M., Ioannidis, P., Klioutchnikov, G., et al. (2017). BUSCO applications from quality assessments to gene prediction and phylogenomics. Mol. Biol. Evol. 35, 543-548. doi: 10.1093/molbev/msx319

Watters, M. K., Randall, T. A., Margolin, B. S., Selker, E. U., and Stadler, D. R. (1999). Action of repeat-induced point mutation on both strands of a duplex and on tandem duplications of various sizes in Neurospora. Genetics 153, $705-714$

Wessa, P. (2020). Free Statistics Software, Office for Research Development and Education, version 1.2.1. Available at: https://www.wessa.net/ (Accessed December 11, 2020).

Wickerham, L. J., Kurtzman, C. P., and Herman, A. I. (1970). Sexual reproduction in Candida lipolytica. Science 167:1141. doi: 10.1126/science.167.3921.1141

Wilken, P. M., Steenkamp, E. T., Wingfield, M. J., De Beer, Z. W., and Wingfield, B. D. (2017). Which MAT gene? Pezizomycotina (Ascomycota) mating-type gene nomenclature reconsidered. Fungal Biol. Rev. 31, 199-211. doi: $10.1016 /$ j.fbr.2017.05.003

Wingfield, B. D., Liu, M., Nguyen, H. D., Lane, F. A., Morgan, S. W., De Vos, L., et al. (2018). Nine draft genome sequences of Claviceps purpurea s. lat., including C. arundinis, C. humidiphila, and C. cf. spartinae, pseudomolecules for the pitch canker pathogen Fusarium circinatum, draft genome of Davidsoniella eucalypti, Grosmannia galeiformis, Quambalaria eucalypti, and Teratosphaeria destructans. IMA Fungus 9:401. doi: 10.5598/imafungus.2018.09.02.10

Wingfield, B. D., Steenkamp, E. T., Santana, Q. C., Coetzee, M., Bam, S., Barnes, I., et al. (2012). First fungal genome sequence from Africa: a preliminary analysis. S. Afr. J. Sci. 108, 104-112. doi: 10.4102/sajs.v108i1/2.537

Wu, C., Kim, Y. -S., Smith, K. M., Li, W., Hood, H. M., Staben, C., et al. (2009). Characterization of chromosome ends in the filamentous fungus Neurospora crassa. Genetics 181, 1129-1145. doi: 10.1534/genetics.107.084392

Wu, L., Wang, M., Zha, G., Zhou, J., Yu, Y., and Lu, H. (2020). Improving the expression of a heterologous protein by genome shuffling in Kluyveromyces marxianus. J. Biotechnol. 320, 11-16. doi: 10.1016/j.jbiotec.2020.06.007

Yilmaz, N., Visagie, C. M., Houbraken, J., Frisvad, J. C., and Samson, R. A. (2014). Polyphasic taxonomy of the genus Talaromyces. Stud. Mycol. 78, 175-341. doi: 10.1016/j.simyco.2014.08.001 
Yin, Z., Ke, X., Li, Z., Chen, J., Gao, X., and Huang, L. (2017). Unconventional recombination in the mating type locus of heterothallic apple canker pathogen Valsa mali. G3 7, 1259-1265. doi: 10.1534/g3.116.037853

Yu, Y., Amich, J., Will, C., Eagle, C. E., Dyer, P. S., and Krappmann, S. (2017). The novel Aspergillus fumigatus MAT1-2-4 mating-type gene is required for mating and cleistothecia formation. Fungal Genet. Biol. 108, 1-12. doi: 10.1016/j.fgb.2017.09.001

Zhang, L., Zhou, Z., Guo, Q., Fokkens, L., Miskei, M., Pócsi, I., et al. (2016). Insights into adaptations to a near-obligate nematode endoparasitic lifestyle from the finished genome of Drechmeria coniospora. Sci. Rep. 6:23122. doi: $10.1038 /$ srep23122
Conflict of Interest: The authors declare that the research was conducted in the absence of any commercial or financial relationships that could be construed as a potential conflict of interest.

Copyright (๑ 2021 van Wyk, Wingfield, De Vos, van der Merwe and Steenkamp. This is an open-access article distributed under the terms of the Creative Commons Attribution License (CC BY). The use, distribution or reproduction in other forums is permitted, provided the original author(s) and the copyright owner(s) are credited and that the original publication in this journal is cited, in accordance with accepted academic practice. No use, distribution or reproduction is permitted which does not comply with these terms. 